\title{
Multi-step hybrid viscosity method for systems of variational inequalities defined over sets of solutions of an equilibrium problem and fixed point problems
}

\author{
Abdul Latif ${ }^{*}$, Lu-Chuan Ceng ${ }^{2}$ and Qamrul Hasan Ansari ${ }^{3}$
}

"Correspondence: alatif@kau.edu.sa 'Department of Mathematics, King Abdulaziz University, Jeddah, 21589, Saudi Arabia

Full list of author information is available at the end of the article

\begin{abstract}
In this paper, we consider a system of variational inequalities defined over the intersection of the set of solutions of an equilibrium problem, the set of common fixed points of a finite family of nonexpansive mappings, and the solution set of a nonexpansive mapping. We also consider a triple hierarchical variational inequality problem, that is, a variational inequality problem defined over a set of solutions of another variational inequality problem which is defined over the intersection of the set of solutions of an equilibrium problem, the set of common fixed points of a finite family of nonexpansive mappings, and the solution set of a nonexpansive mapping. These two problems are very general and include, as special cases, several problems studied in the literature. We propose a multi-step hybrid viscosity method to compute the approximate solutions of our system of variational inequalities and a triple hierarchical variational inequality problem. The convergence analysis of the sequences generated by the proposed method is also studied. In addition, the nontrivial examples of two systems are presented and our results are applied to these examples.

MSC: 49J40; 47H05; 47H19
\end{abstract}

Keywords: multi-step hybrid viscosity method; systems of variational inequalities; equilibrium problems; fixed point problems; convergence analysis

\section{Introduction and formulations}

Let $H$ be a real Hilbert space whose inner product and norm are denoted by $\langle\cdot, \cdot\rangle$ and $\|\cdot\|$, respectively. Let $C$ be a nonempty, closed and convex subset of $H$ and $A: C \rightarrow H$ be a nonlinear mapping. The variational inequality problem (VIP) associated with the set $C$ and the mapping $A$ is stated as follows:

$$
\text { find } x^{*} \in C \text { such that }\left\langle A x^{* \prime}, x-x^{*}\right| \geq 0, \quad \forall x \in C \text {. }
$$

In particular, if $C$ is the set of fixed points of a nonexpansive mapping $T$, denoted by $\operatorname{Fix}(T)$, and if $S$ is another nonexpansive mapping (not necessarily with fixed points), then VIP (1.1) becomes the following problem:

$$
\text { find } x^{\prime \prime} \in \operatorname{Fix}(T) \text { such that }\left\langle(I-S) x^{*}, x-x^{*}\right\rangle \geq 0, \quad \forall x \in \operatorname{Fix}(T) \text {. }
$$

(0) 2012 Latif et al.; licensee Springer. This is an Open Access article distributed under the terms of the Creative Commons Attribution License (http://creativecommons.org/licenses/by/2.0), which permits unrestricted use, distribution, and reproduction in any medium, provided the original work is properly cited. 
It is called a hierarchical variational inequality problem, also known as a hierarchical fixed point problem, and it is studied in [1,2]. Observe that if $S$ has fixed points, then they are the solutions of VIP (1.2). It is worth mentioning that many practical problems can be written in the form of a hierarchical variational inequality problem; see for example [3-5] and the references therein. Such a problem is also a bilevel problem, in which we find a solution of the first problem subject to the condition that its solution is also a fixed point of a mapping. For further details on hierarchical fixed point problems and their applications, we refer to $[1-3,6-18]$ and the references therein. The solution methods presented in $[1,12]$ may not be unique. Therefore, it would be reasonable to identify the unique minimizer of an appropriate objective function over the hierarchical fixed point constraint.

Mainge and Moudafi [2] introduced a hierarchical fixed point approach to finding a solution of VIP (1.2). Subsequently, Moudafi and Mainge [1] studied the explicit scheme for computing a solution of VIP (1.2) by introducing the following iterative algorithm:

$$
x_{n+1}=\lambda_{n} f\left(x_{n}\right)+\left(1-\lambda_{n}\right)\left(\alpha_{n} S x_{n}+\left(1-\alpha_{n}\right) T x_{n}\right) \text {, }
$$

where $f: C \rightarrow C$ and $\left\{\alpha_{n}\right\},\left\{\lambda_{n}\right\} \subset(0,1)$. They also proved the strong convergence of the sequence $\left\{x_{n}\right\}$ generalized by (1.3) to a solution of VIP (1.2).

Yao et al. [19] introduced and analyzed the following two-step iterative algorithm that generates a sequence $\left\{x_{n}\right\}$ by the following explicit scheme:

$$
\left\{\begin{array}{l}
y_{n}=\beta_{n} S x_{n}+\left(1-\beta_{n}\right) x_{n}, \\
x_{n+1}=\alpha_{n} f\left(x_{n}\right)+\left(1-\alpha_{n}\right) T y_{n}, \quad n \geq 1 .
\end{array}\right.
$$

It is easy to see that if $C=\operatorname{Fix}(T)$ and $\Theta(x, y)=\langle(I-S) x, y-x\rangle$, VIP (1.2) can be reformulated as follows:

$$
\text { find } x^{*} \in C \text { such that } \Theta\left(x^{*}, y\right) \geq 0, \quad \forall y \in C \text {. }
$$

It is known as an equilibrium problem. In [20, 21], it is shown that the formulation (1.5) covers monotone inclusion problems, saddle point problems, variational inequality problems, minimization problems, Nash equilibria in noncooperative games, vector equilibrium problems and certain fixed point problems.

Recently, many authors have generalized the classical equilibrium problem introduced in [22] by introducing 'perturbation' to the function $\Theta$. For example, Moudafi [23] studied the following equilibrium problem:

$$
\text { find } x^{*} \in C \text { such that } \Theta\left(x^{*}, y\right)+\left\langle A x^{*}, y-x^{*}\right\rangle \geq 0, \quad \forall y \in C \text {, }
$$

where $A$ is an $\alpha$-inverse strongly monotone operator. In [24-26], the following mixed equilibrium problem is studied:

$$
\text { find } x^{*} \in C \text { such that } \Theta\left(x^{*}, y\right)+\varphi\left(x^{*}\right)-\varphi(y) \geq 0, \quad \forall y \in C \text {, }
$$


where $\varphi: C \rightarrow \mathbb{R}$ is a functional on $C$. Very recently, Marino et al. [27] studied the following equilibrium problem:

$$
\text { find } x^{*} \in C \text { such that } \Theta\left(x^{*}, y\right)+h\left(x^{*}, y\right) \geq 0, \quad \forall y \in C \text {. }
$$

It includes all previous equilibrium problems as special cases. The set of solutions of (1.6) is denoted by $E P(\Theta, h)$.

Lemma 1.1 ([27, Lemma 2.2]) Let $C$ be a nonempty, closed and convex subset of a Hilbert space $H$. Let $\Theta: C \times C \rightarrow \mathbb{R}$ be a bifunction such that

$(\Theta 1) \Theta(x, x)=0, \forall x \in C$

$(\Theta 2) \Theta$ is monotone (that is, $\Theta(x, y)+\Theta(y, x) \leq 0, \forall(x, y) \in C \times C)$ and upper hemicontinuous in the first variable (that is, $\left.\lim _{\sup } \operatorname{su}_{t \rightarrow 0} \Theta(t z+(1-t) x, y) \leq \Theta(x, y), \forall x, y, z \in C\right)$;

$(\Theta 3) \Theta$ is lower semicontinuous and convex in the second variable.

Let $h: C \times C \rightarrow \mathbb{R}$ be a bifunction such that

(h1) $h(x, x)=0, \forall x \in C$;

(h2) $h$ is monotone and weakly upper semicontinuous in the first variable;

(h3) $h$ is convex in the second variable.

\section{Moreover, suppose that}

(H) for fixed $r>0$ and $x \in C$, there exists a bounded $K \subset C$ and $a \in K$ such that for all $z \in C \backslash K,-\Theta(a, z)+h(z, a)+\frac{1}{r}\langle a-z, z-x\rangle<0$.

For $r>0$ and $x \in H$, let $T_{r}: H \rightarrow 2^{C}$ be a mapping defined by

$$
T_{r} x=\left\{z \in C: \Theta(z, y)+h(z, y)+\frac{1}{r}\langle y-z, z-x\rangle \geq 0, \forall y \in C\right\}
$$

(is called the resolvent of $\Theta$ and $h$ ). Then

(i) $T_{r} x \neq \emptyset$;

(ii) $T_{r} x$ is a singleton;

(iii) $T_{r}$ is firmly nonexpansive;

(iv) $E P(\Theta, h)=\operatorname{Fix}\left(T_{r}\right)$ and it is closed and convex;

(v) $($ see $[28])$

$$
\left\|T_{r_{2}} y-T_{r_{1}} x\right\| \leq\|y-x\|+\left|\frac{r_{2}-r_{1}}{r_{2}}\right|\left\|T_{r_{2}} y-y\right\|, \quad \forall x, y \in H, \forall r_{1}, r_{2}>0 .
$$

Marino et al. [27] introduced a multi-step iterative method that generalizes the two-step method studied in [19] from two nonexpansive mappings to a finite family of nonexpansive mappings, and proved that the sequence generated by this method converges strongly to a common fixed point of the mappings which is also a solution of the equilibrium problem (1.6). The multi-step iterative method in [27] involves the Ishikawa-type iterative method and the viscosity approximation method.

On the other hand, by combining the regularization method, the hybrid steepestdescent method, and the projection method, Ceng et al. [29] proposed an iterative algorithm that generates a sequence via the explicit scheme and proved that this sequence converges strongly to a unique solution of the following problem. 
Problem 1.1 Let $F: C \rightarrow H$ be $\kappa$-Lipschitzian and $\eta$-strongly monotone on the nonempty, closed and convex subset $C$ of $H$, where $\kappa$ and $\eta$ are positive constants, that is,

$$
\|F x-F y\| \leq \kappa\|x-y\| \quad \text { and } \quad\langle F x-F y, x-y\rangle \geq \eta\|x-y\|^{2}, \quad \forall x, y \in C .
$$

Let $f: C \rightarrow H$ be a $\rho$-contraction with a coefficient $\rho \in[0,1)$, and $S, T: C \rightarrow C$ be two nonexpansive mappings with $\operatorname{Fix}(T) \neq \emptyset$. Let $0<\mu<2 \eta / \kappa^{2}$ and $0<\gamma \leq \tau$, where $\tau=1-$ $\sqrt{1-\mu\left(2 \eta-\mu \kappa^{2}\right)}$. Then the objective is to find $x^{*} \in \Xi$ such that

$$
\left\langle(\mu F-\gamma f) x^{*}, x-x^{*}\right\rangle \geq 0, \quad \forall x \in \Xi
$$

where $\Xi$ denotes the solution set of the following hierarchical variational inequality problem (HVIP): find $z^{*} \in \operatorname{Fix}(T)$ such that

$$
\left\langle(\mu F-\gamma S) z^{*}, z-z^{*}\right\rangle \geq 0, \quad \forall z \in \operatorname{Fix}(T) .
$$

Since Problem 1.1 has a triple hierarchical structure in contrast with bilevel programming problems (see $[30,31]$ ), that is, a variational inequality problem with a variational inequality constraint over the fixed point set $\operatorname{Fix}(T)$, we also call it a triple hierarchical variational inequality problem, which is a generalization of the triple hierarchical constrained optimization problem (THCOP) considered by liduka [32, 33].

In this paper, we consider the following system of variational inequalities defined over the set consisting of the set of solutions of an equilibrium problem, the set of common fixed points of nonexpansive mappings, and the set of fixed points of a mapping.

Problem 1.2 Let $F: C \rightarrow H$ be $\kappa$-Lipschitzian and $\eta$-strongly monotone on the nonempty, closed and convex subset $C$ of $H, f: C \rightarrow H$ be a $\rho$-contraction with a coefficient $\rho \in[0,1)$ and $S_{i}, S, T: C \rightarrow C$ be nonexpansive mappings for all $i \in\{1, \ldots, N\}$. Assume that $\Theta, h: C \times C \rightarrow \mathbb{R}$ are two bifunctions. Let $0<\mu<2 \eta / \kappa^{2}$ and $0<\gamma \leq \tau$, where $\tau=1-\sqrt{1-\mu\left(2 \eta-\mu \kappa^{2}\right)}$. Then the objective is to find $x^{*} \in \Omega$ such that

$$
\begin{cases}\left\langle(\mu F-\gamma f) x^{*}, x-x^{*}\right\rangle \geq 0, & \forall x \in \Omega, \\ \left\langle(\mu F-\gamma S) x^{*}, y-x^{*}\right\rangle \geq 0, & \forall y \in \Omega,\end{cases}
$$

where $\Omega=\operatorname{Fix}(T) \cap\left(\bigcap_{i} \operatorname{Fix}\left(S_{i}\right)\right) \cap E P(\Theta, h) \neq \emptyset$.

We propose the following multi-step hybrid viscosity method for solving Problem 1.2.

Algorithm 1.1 Let $F: C \rightarrow H$ be $\kappa$-Lipschitzian and $\eta$-strongly monotone on the nonempty, closed and convex subset $C$ of $H, f: C \rightarrow H$ be a $\rho$-contraction with a coefficient $\rho \in[0,1)$ and $S_{i}, S, T: C \rightarrow C$ be nonexpansive mappings for all $i \in\{1, \ldots, N\}$. Assume that $\Theta, h: C \times C \rightarrow \mathbb{R}$ are two bifunctions satisfying the hypotheses of Lemma 1.1. Let $\left\{\lambda_{n}\right\},\left\{\alpha_{n}\right\},\left\{\beta_{n, i}\right\}, i=1, \ldots, N$ be sequences in $(0,1)$ and $\left\{r_{n}\right\}$ be a sequence in $(0, \infty)$ with $\liminf _{n \rightarrow \infty} r_{n}>0$. Let $0<\mu<2 \eta / \kappa^{2}$ and $0<\gamma \leq \tau$, where $\tau=1-\sqrt{1-\mu\left(2 \eta-\mu \kappa^{2}\right)}$. Then the sequence $\left\{x_{n}\right\}$ is generated from an arbitrary initial point $x_{1} \in C$ by the following 
iterative scheme:

$$
\left\{\begin{array}{l}
\Theta\left(u_{n}, y\right)+h\left(u_{n}, y\right)+\frac{1}{r_{n}}\left\langle y-u_{n}, u_{n}-x_{n}\right\rangle \geq 0, \quad \forall y \in C, \\
y_{n, 1}=\beta_{n, 1} S_{1} u_{n}+\left(1-\beta_{n, 1}\right) u_{n}, \\
y_{n, i}=\beta_{n, i} S_{i} u_{n}+\left(1-\beta_{n, i}\right) y_{n, i-1}, \quad i=2, \ldots, N, \\
x_{n+1}=P_{C}\left[\lambda_{n} \gamma\left(\alpha_{n} f\left(x_{n}\right)+\left(1-\alpha_{n}\right) S x_{n}\right)+\left(I-\lambda_{n} \mu F\right) T y_{n, N}\right], \quad n \geq 1 .
\end{array}\right.
$$

In particular, if $f \equiv 0$, then (1.13) reduces to the following iterative scheme:

$$
\left\{\begin{array}{l}
\Theta\left(u_{n}, y\right)+h\left(u_{n}, y\right)+\frac{1}{r_{n}}\left\langle y-u_{n}, u_{n}-x_{n}\right\rangle \geq 0, \quad \forall y \in C, \\
y_{n, 1}=\beta_{n, 1} S_{1} u_{n}+\left(1-\beta_{n, 1}\right) u_{n}, \\
y_{n, i}=\beta_{n, i} S_{i} u_{n}+\left(1-\beta_{n, i}\right) y_{n, i-1}, \quad i=2, \ldots, N, \\
x_{n+1}=P_{C}\left[\lambda_{n}\left(1-\alpha_{n}\right) \gamma S x_{n}+\left(I-\lambda_{n} \mu F\right) T y_{n, N}\right], \quad n \geq 1 .
\end{array}\right.
$$

We prove that, under appropriate conditions, the sequence $\left\{x_{n}\right\}$ generated by Algorithm 1.1 converges strongly to a unique solution of Problem 1.2. In addition, we also consider and study the application of Algorithm 1.1 to solve the following triple hierarchical variational inequality problem (THVIP):

Problem 1.3 Let $F: C \rightarrow H$ be $\kappa$-Lipschitzian and $\eta$-strongly monotone on the nonempty, closed and convex subset $C$ of $H, f: C \rightarrow H$ be a $\rho$-contraction with a coefficient $\rho \in[0,1)$ and $S_{i}, S, T: C \rightarrow C$ be nonexpansive mappings for all $i \in\{1, \ldots, N\}$. Assume that $\Theta, h: C \times C \rightarrow \mathbb{R}$ are two bifunctions. Let $0<\mu<2 \eta / \kappa^{2}$ and $0<\gamma \leq \tau$, where $\tau=1-\sqrt{1-\mu\left(2 \eta-\mu \kappa^{2}\right)}$. Then the objective is to find $x^{*} \in \Xi$ such that

$$
\left\langle(\mu F-\gamma f) x^{*}, x-x^{*}\right\rangle \geq 0, \quad \forall x \in \Xi
$$

where $\Xi$ denotes the solution set of the following hierarchical variational inequality problem (HVIP) of finding $z^{\prime \prime} \in \Omega$ such that

$$
\left\langle(\mu F-\gamma S) z^{*}, z-z^{*}\right\rangle \geq 0, \quad \forall z \in \Omega
$$

where $\Omega=\operatorname{Fix}(T) \cap\left(\bigcap_{i} \operatorname{Fix}\left(S_{i}\right)\right) \cap E P(\Theta, h) \neq \emptyset$.

Moreover, we prove that, under very mild conditions, the sequence $\left\{x_{n}\right\}$ generated by Algorithm 1.1 converges strongly to a unique solution of Problem 1.3. It is worth pointing out that Problem 1.1 is a special case of Problem 1.3 whenever $\Theta \equiv h \equiv 0$ and $S_{i} \equiv I$ for all $i \in\{1, \ldots, N\}$.

\section{Some basic results}

We present here some basic facts and results that are needed in the sequel.

Lemma 2.1 ([34, Lemma 2.1]) Let $\left\{s_{n}\right\}$ be a sequence of nonnegative numbers satisfying the condition

$$
s_{n+1} \leq\left(1-\gamma_{n}\right) s_{n}+\gamma_{n} \delta_{n}, \quad n \geq 1,
$$

where $\left\{\gamma_{n}\right\},\left\{\delta_{n}\right\}$ are the sequences of real numbers such that 
(i) $\left\{\gamma_{n}\right\} \subset[0,1]$ and $\sum_{n=1}^{\infty} \gamma_{n}=\infty$, or equivalently,

$$
\prod_{n=1}^{\infty}\left(1-\gamma_{n}\right):=\lim _{n \rightarrow \infty} \prod_{k=1}^{n}\left(1-\gamma_{k}\right)=0
$$

(ii) $\lim \sup _{n \rightarrow \infty} \delta_{n} \leq 0$, or

(ii) $\sum_{n=1}^{\infty} \gamma_{n} \delta_{n}$ is convergent.

Then $\lim _{n \rightarrow \infty} s_{n}=0$.

Lemma 2.2 ([34, Lemma 3.1]) Let $\lambda$ be a number in $(0,1]$, and let $\mu>0$. Let $F: C \rightarrow H$ be an operator on $C$ such that for some constants $\kappa, \eta>0, F$ is $\kappa$-Lipschitzian and $\eta$-strongly monotone. Associating with a nonexpansive mapping $T: C \rightarrow C$, we define the mapping $T^{\lambda}: C \rightarrow H$ by

$$
T^{\lambda} x:=T x-\lambda \mu F(T x), \quad \forall x \in C
$$

Then $T^{\lambda}$ is a contraction provided $\mu<2 \eta / \kappa^{2}$, that is,

$$
\left\|T^{\lambda} x-T^{\lambda} y\right\| \leq(1-\lambda \tau)\|x-y\|, \quad \forall x, y \in C,
$$

where $\tau=1-\sqrt{1-\mu\left(2 \eta-\mu \kappa^{2}\right)} \in(0,1]$.

In the sequel, given a sequence $\left\{z_{n}\right\}$, we will denote with $\omega_{w}\left(z_{n}\right)$ the set of cluster points of $\left\{z_{n}\right\}$ with respect to the weak topology, that is,

$$
\omega_{w}\left(z_{n}\right)=\left\{z \in H: \text { there exists } n_{k} \rightarrow \infty \text { for which } z_{n_{k}} \rightarrow z\right\} .
$$

Analogously, we denote by $\omega_{s}\left(z_{n}\right)$ the set of cluster points of $\left\{z_{n}\right\}$ with respect to the norm topology, that is,

$$
\omega_{s}\left(z_{n}\right)=\left\{z \in H: \text { there exists } n_{k} \rightarrow \infty \text { for which } z_{n_{k}} \rightarrow z\right\}
$$

Lemma 2.3 ([27, Lemma 2.5]) Suppose that the hypotheses of Lemma 1.1 are satisfied. Let $\left\{r_{n}\right\}$ be a sequence in $(0, \infty)$ with $\liminf _{n \rightarrow \infty} r_{n}>0$. Suppose that $\left\{x_{n}\right\}$ is a bounded sequence. Then the following equivalent statements hold:

(a) If $\left\|x_{n}-T_{r_{n}} x_{n}\right\| \rightarrow 0$, as $n \rightarrow \infty$, every weak cluster point of $\left\{x_{n}\right\}$ solves the problem

$$
\Theta(x, y)+h(x, y) \geq 0, \quad \forall y \in C
$$

that is, $\omega_{w}\left(x_{n}\right) \subseteq E P(\Theta, h)$.

(b) (Demiclosedness principle) If $x_{n} \rightarrow x^{*}$ and $\left\|x_{n}-T_{r_{n}} x_{n}\right\| \rightarrow 0$, as $n \rightarrow \infty$, then $\left(I-T_{r_{k}}\right) x^{*}=0$ for all $k \geq 1$.

Lemma 2.4 ([35]) Let $\left\{\alpha_{n}\right\}$ be a sequence of nonnegative real numbers with $\limsup _{n \rightarrow \infty} \alpha_{n}<\infty$ and $\left\{\beta_{n}\right\}$ be a sequence of real numbers with $\lim _{\sup _{n \rightarrow \infty}} \beta_{n} \leq 0$. Then $\lim \sup _{n \rightarrow \infty} \alpha_{n} \beta_{n} \leq 0$. 


\section{Main results}

Now we present the convergence analysis of Algorithm 1.1 for solving Problem 1.2.

Theorem 3.1 Assume that Problem 1.2 has a solution. Let $\left\{\lambda_{n}\right\},\left\{\alpha_{n}\right\},\left\{\beta_{n, i}\right\}, i=1, \ldots, N$ be sequences in $(0,1)$ such that $\beta_{n, i} \rightarrow \beta_{i} \in(0,1)$ as $n \rightarrow \infty$ for all $i \in\{1, \ldots, N\}$. Suppose that the following conditions hold:

(C1) $0<\liminf _{n \rightarrow \infty} \alpha_{n} \leq \limsup _{n \rightarrow \infty} \alpha_{n}<1$;

(C2) $\lim _{n \rightarrow \infty} \lambda_{n}=0$ and $\sum_{n=1}^{\infty} \lambda_{n}=\infty$;

(C3) $\sum_{n=2}^{\infty}\left|\alpha_{n} \lambda_{n}-\alpha_{n-1} \lambda_{n-1}\right|<\infty$ or $\lim _{n \rightarrow \infty} \frac{\left|\alpha_{n} \lambda_{n}-\alpha_{n-1} \lambda_{n-1}\right|}{\lambda_{n}}=0$;

(C4) $\sum_{n=2}^{\infty}\left|\lambda_{n}-\lambda_{n-1}\right|<\infty$ or $\lim _{n \rightarrow \infty} \frac{\left|\lambda_{n}-\lambda_{n-1}\right|}{\lambda_{n}}=0$;

(C5) $\sum_{n=2}^{\infty}\left|\beta_{n, i}-\beta_{n-1, i}\right|<\infty$ or $\lim _{n \rightarrow \infty} \frac{\left|\beta_{n, i}-\beta_{n-1, i}\right|}{\lambda_{n}}=0$ for all $i \in\{1, \ldots, N\}$;

(C6) $\sum_{n=2}^{\infty}\left|r_{n}-r_{n-1}\right|<\infty$ or $\lim _{n \rightarrow \infty} \frac{\left|r_{n}-r_{n-1}\right|}{\lambda_{n}}=0$.

Then the following assertions hold.

(a) Let $\left\{x_{n}\right\}$ be a sequence generated by the scheme (1.13), then $\left\{x_{n}\right\}$ converges strongly to a unique solution $x^{*} \in \Omega$ of Problem 1.2.

(b) Let $\left\{x_{n}\right\}$ be a sequence generated by the scheme (1.14), then $\left\{x_{n}\right\}$ converges strongly to a unique solution $x^{*} \in \Omega$ of the following system of variational inequalities:

$$
\begin{cases}\left\langle F x^{*}, x-x^{*}\right\rangle \geq 0, & \forall x \in \Omega, \\ \left\langle(\mu F-\gamma S) x^{*}, y-x^{*}\right\rangle \geq 0, & \forall y \in \Omega .\end{cases}
$$

Proof We prove only part (a) since part (b) is a straightforward consequence of part (a). Let $\left\{x_{n}\right\}$ be a sequence generated by the scheme (1.13). First of all, note that $0<\gamma \leq \tau$ and

$$
\begin{aligned}
\mu \eta \geq \tau & \Leftrightarrow \quad \mu \eta \geq 1-\sqrt{1-\mu\left(2 \eta-\mu \kappa^{2}\right)} \\
& \Leftrightarrow \quad \sqrt{1-\mu\left(2 \eta-\mu \kappa^{2}\right)} \geq 1-\mu \eta \\
& \Leftrightarrow \quad 1-2 \mu \eta+\mu^{2} \kappa^{2} \geq 1-2 \mu \eta+\mu^{2} \eta^{2} \\
& \Leftrightarrow \quad \kappa^{2} \geq \eta^{2} \\
& \Leftrightarrow \quad \kappa \geq \eta .
\end{aligned}
$$

Then it follows from the $\rho$-contractiveness of $f$ that

$$
\langle(\mu F-\gamma f) x-(\mu F-\gamma f) y, x-y\rangle \geq(\mu \eta-\gamma \rho)\|x-y\|^{2}, \quad \forall x, y \in C .
$$

Hence, from $\gamma \rho<\gamma \leq \tau \leq \mu \eta$ we deduce that $\mu F-\gamma f$ is $(\mu \eta-\gamma \rho)$-strongly monotone. Since it is clear that $\mu F-\gamma f$ is Lipschitz continuous, there exists a unique solution of the following VIP:

$$
\text { find } x^{*} \in \Omega \text { such that }\left\langle(\mu F-\gamma f) x^{*}, x-x^{*}\right\rangle \geq 0, \quad \forall x \in \Omega \text {. }
$$

Also, since Problem 1.2 has a solution, it is easy to see that Problem 1.2 has a unique solution. In addition, taking into account condition (C1), without loss of generality, we may assume that $\left\{\alpha_{n}\right\} \subset[a, b]$ for some $a, b \in(0,1)$. 
The rest of the proof is divided into several steps.

Step 1 . The sequences $\left\{x_{n}\right\},\left\{y_{n, i}\right\}$ for all $i,\left\{u_{n}\right\}$ are bounded.

Indeed, take a point $v \in \Omega$ arbitrarily. Then by Lemma 1.1, we have from

$$
\left\|y_{n, 1}-v\right\| \leq\left\|u_{n}-v\right\|=\left\|T_{r_{n}} x_{n}-T_{r_{n}} v\right\| \leq\left\|x_{n}-v\right\| .
$$

For all from $i=2$ to $i=N$, by induction, one proves that

$$
\left\|y_{n, i}-v\right\| \leq \beta_{n, i}\left\|u_{n}-v\right\|+\left(1-\beta_{n, i}\right)\left\|y_{n, i-1}-v\right\| \leq\left\|u_{n}-v\right\| \leq\left\|x_{n}-v\right\| .
$$

Hence, we obtain that for all $i \in\{1, \ldots, N\}$

$$
\left\|y_{n, i}-v\right\| \leq\left\|u_{n}-v\right\| \leq\left\|x_{n}-v\right\| .
$$

Also, utilizing Lemma 2.2 and (1.13), we have

$$
\begin{aligned}
& \left\|x_{n+1}-v\right\| \\
& =\left\|P_{C}\left[\lambda_{n} \gamma\left(\alpha_{n} f\left(x_{n}\right)+\left(1-\alpha_{n}\right) S x_{n}\right)+\left(I-\lambda_{n} \mu F\right) T y_{n, N}\right]-P_{C} v\right\| \\
& \leq\left\|\lambda_{n} \gamma\left(\alpha_{n} f\left(x_{n}\right)+\left(1-\alpha_{n}\right) S x_{n}\right)+\left(I-\lambda_{n} \mu F\right) T y_{n, N}-v\right\| \\
& =\| \lambda_{n} \gamma\left(\alpha_{n} f\left(x_{n}\right)+\left(1-\alpha_{n}\right) S x_{n}\right)-\lambda_{n} \mu F T \nu \\
& +\left(I-\lambda_{n} \mu F\right) T y_{n, N}-\left(I-\lambda_{n} \mu F\right) T v \| \\
& \leq\left\|\lambda_{n} \gamma\left(\alpha_{n} f\left(x_{n}\right)+\left(1-\alpha_{n}\right) S x_{n}\right)-\lambda_{n} \mu F T \nu\right\| \\
& +\left\|\left(I-\lambda_{n} \mu F\right) T y_{n, N}-\left(I-\lambda_{n} \mu F\right) T v\right\| \\
& =\lambda_{n}\left\|\alpha_{n}\left(\gamma f\left(x_{n}\right)-\mu F v\right)+\left(1-\alpha_{n}\right)\left(\gamma S x_{n}-\mu F v\right)\right\| \\
& +\left\|\left(I-\lambda_{n} \mu F\right) T y_{n, N}-\left(I-\lambda_{n} \mu F\right) T v\right\| \\
& \leq \lambda_{n}\left[\alpha_{n}\left\|\gamma f\left(x_{n}\right)-\mu F v\right\|+\left(1-\alpha_{n}\right)\left\|\gamma S x_{n}-\mu F v\right\|\right] \\
& +\left(1-\lambda_{n} \tau\right)\left\|y_{n, N}-v\right\| \\
& \leq \lambda_{n}\left[\alpha_{n}\left(\left\|\gamma f\left(x_{n}\right)-\gamma f(v)\right\|+\|\gamma f(v)-\mu F v\|\right)\right. \\
& \left.+\left(1-\alpha_{n}\right)\left(\left\|\gamma S x_{n}-\gamma S v\right\|+\|\gamma S v-\mu F v\|\right)\right] \\
& +\left(1-\lambda_{n} \tau\right)\left\|y_{n, N}-v\right\| \\
& \leq \lambda_{n}\left[\alpha_{n} \gamma \rho\left\|x_{n}-v\right\|+\alpha_{n}\|\gamma f(v)-\mu F v\|+\left(1-\alpha_{n}\right) \gamma\left\|x_{n}-v\right\|\right. \\
& \left.+\left(1-\alpha_{n}\right)\|\gamma S v-\mu F v\|\right]+\left(1-\lambda_{n} \tau\right)\left\|x_{n}-v\right\| \\
& \leq \lambda_{n}\left[\gamma\left(1-\alpha_{n}(1-\rho)\right)\left\|x_{n}-v\right\|+\max \{\|\gamma f(v)-\mu F v\|,\|\gamma S v-\mu F v\|\}\right] \\
& +\left(1-\lambda_{n} \tau\right)\left\|x_{n}-v\right\| \\
& \leq\left(1-\lambda_{n} \gamma \alpha_{n}(1-\rho)\right)\left\|x_{n}-v\right\|+\lambda_{n} \max \{\|\gamma f(v)-\mu F v\|,\|\gamma S v-\mu F v\|\} \\
& \leq\left(1-\lambda_{n} \gamma a(1-\rho)\right)\left\|x_{n}-v\right\|+\lambda_{n} \max \{\|\gamma f(v)-\mu F v\|,\|\gamma S v-\mu F v\|\},
\end{aligned}
$$


due to $0<\gamma \leq \tau$. So, calling

$$
M=\max \left\{\left\|x_{1}-v\right\|, \frac{\|\gamma f(v)-\mu F v\|}{\gamma a(1-\rho)}, \frac{\|\gamma S v-\mu F v\|}{\gamma a(1-\rho)}\right\},
$$

by induction we derive $\left\|x_{n}-v\right\| \leq M$ for all $n \geq 1$. We obtain the claim.

Step 2 . $\lim _{n \rightarrow \infty}\left\|x_{n+1}-x_{n}\right\|=0$, that is, $\left\{x_{n}\right\}$ is asymptotically regular. Indeed, for each $n \geq 1$, we set

$$
z_{n}=\lambda_{n} \gamma\left(\alpha_{n} f\left(x_{n}\right)+\left(1-\alpha_{n}\right) S x_{n}\right)+\left(I-\lambda_{n} \mu F\right) T y_{n, N}
$$

Then we observe that

$$
\begin{aligned}
z_{n}-z_{n-1}= & \alpha_{n} \lambda_{n} \gamma\left[f\left(x_{n}\right)-f\left(x_{n-1}\right)\right]+\lambda_{n}\left(1-\alpha_{n}\right) \gamma\left(S x_{n}-S x_{n-1}\right) \\
& +\left[\left(I-\lambda_{n} \mu F\right) T y_{n, N}-\left(I-\lambda_{n} \mu F\right) T y_{n-1, N}\right] \\
& +\left(\alpha_{n} \lambda_{n}-\alpha_{n-1} \lambda_{n-1}\right) \gamma\left[f\left(x_{n-1}\right)-S x_{n-1}\right] \\
& +\left(\lambda_{n}-\lambda_{n-1}\right)\left(\gamma S x_{n-1}-\mu F T y_{n-1, N}\right) .
\end{aligned}
$$

Let $M_{0}>0$ be a constant such that

$$
\sup _{n \geq 1}\left\{\gamma\left\|f\left(x_{n}\right)-S x_{n}\right\|+\left\|\gamma S x_{n}-\mu F T y_{n, N}\right\|\right\} \leq M_{0} .
$$

It follows from (1.13) and (3.2) that

$$
\begin{aligned}
\left\|x_{n+1}-x_{n}\right\| & \left\|P_{C} z_{n}-P_{C} z_{n-1}\right\| \\
\leq & \left\|z_{n}-z_{n-1}\right\| \\
\leq & \alpha_{n} \lambda_{n} \gamma\left\|f\left(x_{n}\right)-f\left(x_{n-1}\right)\right\|+\lambda_{n}\left(1-\alpha_{n}\right) \gamma\left\|S x_{n}-S x_{n-1}\right\| \\
& +\left\|\left(I-\lambda_{n} \mu F\right) T y_{n, N}-\left(I-\lambda_{n} \mu F\right) T y_{n-1, N}\right\| \\
& +\left|\alpha_{n} \lambda_{n}-\alpha_{n-1} \lambda_{n-1}\right| \gamma\left\|f\left(x_{n-1}\right)-S x_{n-1}\right\| \\
& +\left|\lambda_{n}-\lambda_{n-1}\right|\left\|\gamma S x_{n-1}-\mu F T y_{n-1, N}\right\| \\
\leq & \alpha_{n} \lambda_{n} \gamma \rho\left\|x_{n}-x_{n-1}\right\|+\lambda_{n}\left(1-\alpha_{n}\right) \gamma\left\|x_{n}-x_{n-1}\right\| \\
& +\left(1-\lambda_{n} \tau\right)\left\|y_{n, N}-y_{n-1, N}\right\| \\
& +\left|\alpha_{n} \lambda_{n}-\alpha_{n-1} \lambda_{n-1}\right| M_{0}+\left|\lambda_{n}-\lambda_{n-1}\right| M_{0} \\
= & \lambda_{n}\left(1-\alpha_{n}(1-\rho)\right) \gamma\left\|x_{n}-x_{n-1}\right\|+\left(1-\lambda_{n} \tau\right)\left\|y_{n, N}-y_{n-1, N}\right\| \\
& +\left[\left|\alpha_{n} \lambda_{n}-\alpha_{n-1} \lambda_{n-1}\right|+\left|\lambda_{n}-\lambda_{n-1}\right|\right] M_{0} \\
\leq & \lambda_{n} \gamma(1-a(1-\rho))\left\|x_{n}-x_{n-1}\right\|+\left(1-\lambda_{n} \tau\right)\left\|y_{n, N}-y_{n-1, N}\right\| \\
& +\left[\left|\alpha_{n} \lambda_{n}-\alpha_{n-1} \lambda_{n-1}\right|+\left|\lambda_{n}-\lambda_{n-1}\right|\right] M_{0} .
\end{aligned}
$$


By the definition of $y_{n, i}$, we obtain that for all $i=N, \ldots, 2$

$$
\begin{aligned}
\left\|y_{n, i}-y_{n-1, i}\right\| \leq & \beta_{n, i}\left\|u_{n}-u_{n-1}\right\|+\left\|S_{i} u_{n-1}-y_{n-1, i-1}\right\|\left|\beta_{n, i}-\beta_{n-1, i}\right| \\
& +\left(1-\beta_{n, i}\right)\left\|y_{n, i-1}-y_{n-1, i-1}\right\| .
\end{aligned}
$$

In the case $i=1$, we have

$$
\begin{aligned}
\left\|y_{n, 1}-y_{n-1,1}\right\| \leq & \beta_{n, 1}\left\|u_{n}-u_{n-1}\right\| \\
& \quad+\left\|S_{1} u_{n-1}-u_{n-1}\right\|\left|\beta_{n, 1}-\beta_{n-1,1}\right|+\left(1-\beta_{n, 1}\right)\left\|u_{n}-u_{n-1}\right\| \\
= & \left\|u_{n}-u_{n-1}\right\|+\left\|S_{1} u_{n-1}-u_{n-1}\right\|\left|\beta_{n, 1}-\beta_{n-1,1}\right| .
\end{aligned}
$$

Substituting (3.5) in all (3.4)-type inequalities, we obtain that for $i=2, \ldots, N$

$$
\begin{gathered}
\left\|y_{n, i}-y_{n-1, i}\right\| \leq\left\|u_{n}-u_{n-1}\right\|+\sum_{k=2}^{i}\left\|S_{k} u_{n-1}-y_{n-1, k-1}\right\|\left|\beta_{n, k}-\beta_{n-1, k}\right| \\
+\left\|S_{1} u_{n-1}-u_{n-1}\right\|\left|\beta_{n, 1}-\beta_{n-1,1}\right| .
\end{gathered}
$$

So, we conclude that

$$
\begin{aligned}
& \left\|x_{n+1}-x_{n}\right\| \\
& \leq \lambda_{n} \gamma(1-a(1-\rho))\left\|x_{n}-x_{n-1}\right\|+\left(1-\lambda_{n} \tau\right)\left\|y_{n, N}-y_{n-1, N}\right\| \\
& \quad+\left[\left|\alpha_{n} \lambda_{n}-\alpha_{n-1} \lambda_{n-1}\right|+\left|\lambda_{n}-\lambda_{n-1}\right|\right] M_{0} \\
& \leq \lambda_{n} \gamma(1-a(1-\rho))\left\|x_{n}-x_{n-1}\right\|+\left[\left|\alpha_{n} \lambda_{n}-\alpha_{n-1} \lambda_{n-1}\right|+\left|\lambda_{n}-\lambda_{n-1}\right|\right] M_{0} \\
& \quad+\left(1-\lambda_{n} \tau\right)\left\|u_{n}-u_{n-1}\right\|+\sum_{k=2}^{N}\left\|S_{k} u_{n-1}-y_{n-1, k-1}\right\|\left|\beta_{n, k}-\beta_{n-1, k}\right| \\
& \quad+\left\|S_{1} u_{n-1}-u_{n-1}\right\|\left|\beta_{n, 1}-\beta_{n-1,1}\right| .
\end{aligned}
$$

By Lemma 1.1(v), we know that

$$
\left\|u_{n}-u_{n-1}\right\| \leq\left\|x_{n}-x_{n-1}\right\|+L\left|1-\frac{r_{n-1}}{r_{n}}\right|
$$

where $L=\sup _{n \geq 1}\left\|u_{n}-x_{n}\right\|$. So, substituting (3.6) in the last inequality, we obtain

$$
\begin{aligned}
& \left\|x_{n+1}-x_{n}\right\| \\
& \leq \lambda_{n} \gamma(1-a(1-\rho))\left\|x_{n}-x_{n-1}\right\|+\left[\left|\alpha_{n} \lambda_{n}-\alpha_{n-1} \lambda_{n-1}\right|+\left|\lambda_{n}-\lambda_{n-1}\right|\right] M_{0} \\
& \quad+\left(1-\lambda_{n} \tau\right)\left\|x_{n}-x_{n-1}\right\|+L\left|\frac{r_{n}-r_{n-1}}{r_{n}}\right|+\sum_{k=2}^{N}\left\|S_{k} u_{n-1}-y_{n-1, k-1}\right\|\left|\beta_{n, k}-\beta_{n-1, k}\right| \\
& \quad+\left\|S_{1} u_{n-1}-u_{n-1}\right\|\left|\beta_{n, 1}-\beta_{n-1,1}\right| .
\end{aligned}
$$

If we call

$$
M_{1}:=\max \left\{M_{0}, L, \sup _{n \geq 2, i=2, \ldots, N}\left\|S_{i} u_{n-1}-y_{n-1, i-1}\right\|, \sup _{n \geq 2}\left\|S_{1} u_{n-1}-u_{n-1}\right\|\right\}
$$


and $c>0$ a minorant for $\left\{r_{n}\right\}$, we have

$$
\begin{aligned}
\left\|x_{n+1}-x_{n}\right\| \leq & \left(1-\lambda_{n} \gamma a(1-\rho)\right)\left\|x_{n}-x_{n-1}\right\| \\
& +M_{1}\left[\left|\alpha_{n} \lambda_{n}-\alpha_{n-1} \lambda_{n-1}\right|+\left|\lambda_{n}-\lambda_{n-1}\right|\right. \\
& \left.+\frac{\left|r_{n}-r_{n-1}\right|}{c}+\sum_{k=1}^{N}\left|\beta_{n, k}-\beta_{n-1, k}\right|\right],
\end{aligned}
$$

due to $0<\gamma \leq \tau$. By conditions (C2)-(C6) and Lemma 2.1, we obtain the claim.

Step 3. $\left\|x_{n}-u_{n}\right\|=\left\|x_{n}-T_{r_{n}} x_{n}\right\| \rightarrow 0$ as $n \rightarrow \infty$.

Indeed, by the firm nonexpansivity of $T_{r_{n}}$, a standard calculation (see [36]) shows that for all $p \in E P(\Theta, h)$

$$
\left\|u_{n}-p\right\|^{2} \leq\left\|x_{n}-p\right\|^{2}-\left\|x_{n}-u_{n}\right\|^{2} .
$$

So, take a point $v \in \Omega$ arbitrarily; then utilizing Lemma 2.2 and (1.13), we have

$$
\begin{aligned}
&\left\|x_{n+1}-v\right\|^{2} \\
& \leq\left\|\lambda_{n} \gamma\left(\alpha_{n} f\left(x_{n}\right)+\left(1-\alpha_{n}\right) S x_{n}\right)+\left(I-\lambda_{n} \mu F\right) T y_{n, N}-v\right\|^{2} \\
&= \| \lambda_{n} \gamma\left(\alpha_{n} f\left(x_{n}\right)+\left(1-\alpha_{n}\right) S x_{n}\right)-\lambda_{n} \mu F T v \\
&+\left(I-\lambda_{n} \mu F\right) T y_{n, N}-\left(I-\lambda_{n} \mu F\right) T v \|^{2} \\
& \leq\left\{\left\|\lambda_{n} \gamma\left(\alpha_{n} f\left(x_{n}\right)+\left(1-\alpha_{n}\right) S x_{n}\right)-\lambda_{n} \mu F T v\right\|\right. \\
&\left.+\left\|\left(I-\lambda_{n} \mu F\right) T y_{n, N}-\left(I-\lambda_{n} \mu F\right) T v\right\|\right\}^{2} \\
& \leq\left\{\lambda_{n}\left\|\alpha_{n}\left(\gamma f\left(x_{n}\right)-\mu F v\right)+\left(1-\alpha_{n}\right)\left(\gamma S x_{n}-\mu F v\right)\right\|\right. \\
&\left.+\left(1-\lambda_{n} \tau\right)\left\|y_{n, N}-v\right\|\right\}^{2} \\
& \leq \lambda_{n} \frac{1}{\tau}\left[\alpha_{n}\left\|\gamma f\left(x_{n}\right)-\mu F v\right\|+\left(1-\alpha_{n}\right)\left\|\gamma S x_{n}-\mu F v\right\|\right]^{2} \\
&+\left(1-\lambda_{n} \tau\right)\left\|y_{n, N}-v\right\|^{2} \\
& \leq \lambda_{n} \frac{1}{\tau}\left[\left\|\gamma f\left(x_{n}\right)-\mu F v\right\|+\left\|\gamma S x_{n}-\mu F v\right\|\right]^{2}+\left(1-\lambda_{n} \tau\right)\left\|u_{n}-v\right\|^{2} \\
& \leq \lambda_{n} \frac{1}{\tau}\left[\left\|\gamma f\left(x_{n}\right)-\mu F v\right\|+\left\|\gamma S x_{n}-\mu F v\right\|\right]^{2} \\
&+\left(1-\lambda_{n} \tau\right)\left\|x_{n}-v\right\|^{2}-\left(1-\lambda_{n} \tau\right)\left\|x_{n}-u_{n}\right\|^{2} .
\end{aligned}
$$

This implies that

$$
\begin{aligned}
(1 & \left.-\lambda_{n} \tau\right)\left\|x_{n}-u_{n}\right\|^{2} \\
& \leq \lambda_{n} \frac{1}{\tau}\left[\left\|\gamma f\left(x_{n}\right)-\mu F v\right\|+\left\|\gamma S x_{n}-\mu F v\right\|\right]^{2}+\left(1-\lambda_{n} \tau\right)\left\|x_{n}-v\right\|^{2}-\left\|x_{n+1}-v\right\|^{2} \\
& \leq \lambda_{n} \frac{1}{\tau}\left[\left\|\gamma f\left(x_{n}\right)-\mu F v\right\|+\left\|\gamma S x_{n}-\mu F v\right\|\right]^{2}+\left\|x_{n}-v\right\|^{2}-\left\|x_{n+1}-v\right\|^{2} \\
& \leq \lambda_{n} \frac{1}{\tau}\left[\left\|\gamma f\left(x_{n}\right)-\mu F v\right\|+\left\|\gamma S x_{n}-\mu F v\right\|\right]^{2}+\left\|x_{n+1}-x_{n}\right\|\left(\left\|x_{n}-v\right\|+\left\|x_{n+1}-v\right\|\right) .
\end{aligned}
$$


Since $\left\|x_{n+1}-x_{n}\right\| \rightarrow 0$ and $\lambda_{n} \rightarrow 0$ as $n \rightarrow \infty$, by the boundedness of $\left\{x_{n}\right\}$ we conclude that $\lim _{n \rightarrow \infty}\left\|x_{n}-u_{n}\right\|=0$.

Step 4. For all $i \in\{1, \ldots, N\},\left\|S_{i} u_{n}-u_{n}\right\| \rightarrow 0$ as $n \rightarrow \infty$.

Indeed, let us show that for every $i \in\{1, \ldots, N\}$ one has $\left\|S_{i} u_{n}-y_{n, i-1}\right\| \rightarrow 0$ as $n \rightarrow \infty$. Take a point $v \in \Omega$ arbitrarily. When $i=N$, utilizing Lemmas 2.3 and 2.4, and (1.13), we have

$$
\begin{aligned}
\| x_{n+1}- & v \|^{2} \\
\leq & \left\|\lambda_{n} \gamma\left(\alpha_{n} f\left(x_{n}\right)+\left(1-\alpha_{n}\right) S x_{n}\right)+\left(I-\lambda_{n} \mu F\right) T y_{n, N}-v\right\|^{2} \\
= & \| \lambda_{n} \gamma\left(\alpha_{n} f\left(x_{n}\right)+\left(1-\alpha_{n}\right) S x_{n}\right)-\lambda_{n} \mu F T v \\
& +\left(I-\lambda_{n} \mu F\right) T y_{n, N}-\left(I-\lambda_{n} \mu F\right) T v \|^{2} \\
\leq & \left\{\left\|\lambda_{n} \gamma\left(\alpha_{n} f\left(x_{n}\right)+\left(1-\alpha_{n}\right) S x_{n}\right)-\lambda_{n} \mu F T v\right\|\right. \\
& \left.+\left\|\left(I-\lambda_{n} \mu F\right) T y_{n, N}-\left(I-\lambda_{n} \mu F\right) T v\right\|\right\}^{2} \\
\leq & \left\{\lambda_{n}\left\|\alpha_{n}\left(\gamma f\left(x_{n}\right)-\mu F v\right)+\left(1-\alpha_{n}\right)\left(\gamma S x_{n}-\mu F v\right)\right\|\right. \\
& \left.+\left(1-\lambda_{n} \tau\right)\left\|y_{n, N}-v\right\|\right\}^{2} \\
\leq & \lambda_{n} \frac{1}{\tau}\left[\left\|\gamma f\left(x_{n}\right)-\mu F v\right\|+\left\|\gamma S x_{n}-\mu F v\right\|\right]^{2} \\
& +\left(1-\lambda_{n} \tau\right)\left\|y_{n, N}-v\right\|^{2} \\
= & \lambda_{n} \frac{1}{\tau}\left[\left\|\gamma f\left(x_{n}\right)-\mu F v\right\|+\left\|\gamma S x_{n}-\mu F v\right\|\right]^{2} \\
& +\left(1-\lambda_{n} \tau\right) \beta_{n, N}\left\|S_{N} u_{n}-v\right\|^{2} \\
& +\left(1-\lambda_{n} \tau\right)\left(1-\beta_{n, N}\right)\left\|y_{n, N-1}-v\right\|^{2} \\
& -\left(1-\lambda_{n} \tau\right)\left(1-\beta_{n, N}\right) \beta_{n, N}\left\|S_{N} u_{n}-y_{n, N-1}\right\|^{2} \\
\leq & \lambda_{n} \frac{1}{\tau}\left[\left\|\gamma f\left(x_{n}\right)-\mu F v\right\|+\left\|\gamma S x_{n}-\mu F v\right\|\right]^{2} \\
& +\left(1-\lambda_{n} \tau\right)\left\|u_{n}-v\right\|^{2} \\
& -\left(1-\lambda_{n} \tau\right) \beta_{n, N}\left(1-\beta_{n, N}\right)\left\|S_{N} u_{n}-y_{n, N-1}\right\|^{2} \\
\leq & \lambda_{n} \frac{1}{\tau}\left[\left\|\gamma f\left(x_{n}\right)-\mu F v\right\|+\left\|\gamma S x_{n}-\mu F v\right\|\right]^{2}+\left\|x_{n}-v\right\|^{2} \\
& -\left(1-\lambda_{n} \tau\right) \beta_{n, N}\left(1-\beta_{n, N}\right)\left\|S_{N} u_{n}-y_{n, N-1}\right\|^{2} . \\
&
\end{aligned}
$$

So, we have

$$
\begin{aligned}
& \left(1-\lambda_{n} \tau\right) \beta_{n, N}\left(1-\beta_{n, N}\right)\left\|S_{N} u_{n}-y_{n, N-1}\right\|^{2} \\
& \quad \leq \lambda_{n} \frac{1}{\tau}\left[\left\|\gamma f\left(x_{n}\right)-\mu F v\right\|+\left\|\gamma S x_{n}-\mu F v\right\|\right]^{2}+\left\|x_{n}-v\right\|^{2}-\left\|x_{n+1}-v\right\|^{2} \\
& \quad \leq \lambda_{n} \frac{1}{\tau}\left[\left\|\gamma f\left(x_{n}\right)-\mu F v\right\|+\left\|\gamma S x_{n}-\mu F v\right\|\right]^{2}+\left\|x_{n+1}-x_{n}\right\|\left(\left\|x_{n}-v\right\|+\left\|x_{n+1}-v\right\|\right) .
\end{aligned}
$$

Since $\beta_{n, N} \rightarrow \beta_{N} \in(0,1),\left\|x_{n+1}-x_{n}\right\| \rightarrow 0$ and $\lambda_{n} \rightarrow 0$ as $n \rightarrow \infty$, by the boundedness of $\left\{x_{n}\right\}$ we conclude that $\lim _{n \rightarrow \infty}\left\|S_{N} u_{n}-y_{n, N-1}\right\|=0$. 
Take $i \in\{1, \ldots, N-1\}$ arbitrarily. Then, we have

$$
\begin{aligned}
\left\|x_{n+1}-v\right\|^{2} \\
\leq \lambda_{n} \frac{1}{\tau}\left[\left\|\gamma f\left(x_{n}\right)-\mu F v\right\|+\left\|\gamma S x_{n}-\mu F v\right\|\right]^{2}+\left(1-\lambda_{n} \tau\right)\left\|y_{n, N}-v\right\|^{2} \\
\leq \lambda_{n} \frac{1}{\tau}\left[\left\|\gamma f\left(x_{n}\right)-\mu F v\right\|+\left\|\gamma S x_{n}-\mu F v\right\|\right]^{2} \\
\quad+\left(1-\lambda_{n} \tau\right)\left[\beta_{n, N}\left\|S_{N} u_{n}-v\right\|^{2}+\left(1-\beta_{n, N}\right)\left\|y_{n, N-1}-v\right\|^{2}\right] \\
\leq \lambda_{n} \frac{1}{\tau}\left[\left\|\gamma f\left(x_{n}\right)-\mu F v\right\|+\left\|\gamma S x_{n}-\mu F v\right\|\right]^{2} \\
\quad+\left(1-\lambda_{n} \tau\right) \beta_{n, N}\left\|x_{n}-v\right\|^{2}+\left(1-\lambda_{n} \tau\right)\left(1-\beta_{n, N}\right)\left\|y_{n, N-1}-v\right\|^{2} \\
\leq \lambda_{n} \frac{1}{\tau}\left[\left\|\gamma f\left(x_{n}\right)-\mu F v\right\|+\left\|\gamma S x_{n}-\mu F v\right\|\right]^{2}+\left(1-\lambda_{n} \tau\right) \beta_{n, N}\left\|x_{n}-v\right\|^{2} \\
\quad+\left(1-\lambda_{n} \tau\right)\left(1-\beta_{n, N}\right)\left[\beta_{n, N-1}\left\|S_{N-1} u_{n}-v\right\|^{2}+\left(1-\beta_{n, N-1}\right)\left\|y_{n, N-2}-v\right\|^{2}\right] \\
\leq \lambda_{n} \frac{1}{\tau}\left[\left\|\gamma f\left(x_{n}\right)-\mu F v\right\|+\left\|\gamma S x_{n}-\mu F v\right\|\right]^{2} \\
\quad+\left(1-\lambda_{n} \tau\right)\left(\beta_{n, N}+\left(1-\beta_{n, N}\right) \beta_{n, N-1}\right)\left\|x_{n}-v\right\|^{2} \\
\quad+\left(1-\lambda_{n} \tau\right) \prod_{k=N-1}^{N}\left(1-\beta_{n, k}\right)\left\|y_{n, N-2}-v\right\|^{2}
\end{aligned}
$$

and so, after $(N-i+1)$-iterations,

$$
\begin{aligned}
\| x_{n+1}- & v \|^{2} \\
\leq & \lambda_{n} \frac{1}{\tau}\left[\left\|\gamma f\left(x_{n}\right)-\mu F v\right\|+\left\|\gamma S x_{n}-\mu F v\right\|\right]^{2} \\
& +\left(1-\lambda_{n} \tau\right)\left(\beta_{n, N}+\sum_{j=i+2}^{N}\left(\prod_{p=j}^{N}\left(1-\beta_{n, p}\right)\right) \beta_{n, j-1}\right) \\
& \times\left\|x_{n}-v\right\|^{2}+\left(1-\lambda_{n} \tau\right) \prod_{k=i+1}^{N}\left(1-\beta_{n, k}\right)\left\|y_{n, i}-v\right\|^{2} \\
\leq & \lambda_{n} \frac{1}{\tau}\left[\left\|\gamma f\left(x_{n}\right)-\mu F v\right\|+\left\|\gamma S x_{n}-\mu F v\right\|\right]^{2} \\
& +\left(1-\lambda_{n} \tau\right)\left(\beta_{n, N}+\sum_{j=i+2}^{N}\left(\prod_{p=j}^{N}\left(1-\beta_{n, p}\right)\right) \beta_{n, j-1}\right) \\
& \times\left\|x_{n}-v\right\|^{2}+\left(1-\lambda_{n} \tau\right) \prod_{k=i+1}^{N}\left(1-\beta_{n, k}\right) \\
& \times\left[\beta_{n, i}\left\|S_{i} u_{n}-v\right\|^{2}+\left(1-\beta_{n, i}\right)\left\|y_{n, i-1}-v\right\|^{2}\right. \\
& \left.-\beta_{n, i}\left(1-\beta_{n, i}\right)\left\|S_{i} u_{n}-y_{n, i-1}\right\|^{2}\right] \\
\leq & \lambda_{n} \frac{1}{\tau}\left[\left\|\gamma f\left(x_{n}\right)-\mu F v\right\|+\left\|\gamma S x_{n}-\mu F v\right\|\right]^{2}+\left(1-\lambda_{n} \tau\right)\left\|x_{n}-v\right\|^{2} \\
& -\beta_{n, i}\left(1-\lambda_{n} \tau\right) \prod_{k=i}^{N}\left(1-\beta_{n, k}\right)\left\|S_{i} u_{n}-y_{n, i-1}\right\|^{2} .
\end{aligned}
$$


Again, we obtain

$$
\begin{aligned}
& \left(1-\lambda_{n} \tau\right) \beta_{n, i} \prod_{k=i}^{N}\left(1-\beta_{n, k}\right)\left\|S_{i} u_{n}-y_{n, i-1}\right\|^{2} \\
& \quad \leq \lambda_{n} \frac{1}{\tau}\left[\left\|\gamma f\left(x_{n}\right)-\mu F v\right\|+\left\|\gamma S x_{n}-\mu F v\right\|\right]^{2}+\left\|x_{n}-v\right\|^{2}-\left\|x_{n+1}-v\right\|^{2} \\
& \quad \leq \lambda_{n} \frac{1}{\tau}\left[\left\|\gamma f\left(x_{n}\right)-\mu F v\right\|+\left\|\gamma S x_{n}-\mu F v\right\|\right]^{2}+\left\|x_{n+1}-x_{n}\right\|\left(\left\|x_{n}-v\right\|+\left\|x_{n+1}-v\right\|\right) .
\end{aligned}
$$

Since for all $k \in\{1, \ldots, N\}, \beta_{n, k} \rightarrow \beta_{k} \in(0,1),\left\|x_{n+1}-x_{n}\right\| \rightarrow 0$ and $\lambda_{n} \rightarrow 0$ as $n \rightarrow \infty$, by the boundedness of $\left\{x_{n}\right\}$ we conclude that

$$
\lim _{n \rightarrow \infty}\left\|S_{i} u_{n}-y_{n, i-1}\right\|=0
$$

Obviously, for $i=1$ we have $\lim _{n \rightarrow \infty}\left\|S_{1} u_{n}-u_{n}\right\|=0$. To conclude, we have that

$$
\left\|S_{2} u_{n}-u_{n}\right\| \leq\left\|S_{2} u_{n}-y_{n, 1}\right\|+\left\|y_{n, 1}-u_{n}\right\|=\left\|S_{2} u_{n}-y_{n, 1}\right\|+\beta_{n, 1}\left\|S_{1} u_{n}-u_{n}\right\|,
$$

which hence implies that $\lim _{n \rightarrow \infty}\left\|S_{2} u_{n}-u_{n}\right\|=0$. Consequently, by induction, we get $\lim _{n \rightarrow \infty}\left\|S_{i} u_{n}-u_{n}\right\|=0$ for all $i=2, \ldots, N$ since it is enough to observe that

$$
\begin{aligned}
\left\|S_{i} u_{n}-u_{n}\right\| & \leq\left\|S_{i} u_{n}-y_{n, i-1}\right\|+\left\|y_{n, i-1}-S_{i-1} u_{n}\right\|+\left\|S_{i-1} u_{n}-u_{n}\right\| \\
& \leq\left\|S_{i} u_{n}-y_{n, i-1}\right\|+\left(1-\beta_{n, i-1}\right)\left\|S_{i-1} u_{n}-y_{n, i-2}\right\|+\left\|S_{i-1} u_{n}-u_{n}\right\| .
\end{aligned}
$$

Step 5. $\lim _{n \rightarrow \infty}\left\|y_{n, N}-x_{n}\right\|=\lim _{n \rightarrow \infty}\left\|x_{n}-T x_{n}\right\|=0$ and $\omega_{w}\left(x_{n}\right) \subset \Omega$.

Indeed, since $\left\|x_{n}-u_{n}\right\| \rightarrow 0$ as $n \rightarrow \infty$, we have $\omega_{w}\left(x_{n}\right)=\omega_{w}\left(u_{n}\right)$ and $\omega_{s}\left(x_{n}\right)=\omega_{s}\left(u_{n}\right)$.

Now, we observe that

$$
\left\|x_{n}-y_{n, 1}\right\| \leq\left\|x_{n}-u_{n}\right\|+\left\|y_{n, 1}-u_{n}\right\|=\left\|x_{n}-u_{n}\right\|+\beta_{n, 1}\left\|S_{1} u_{n}-u_{n}\right\|
$$

By Step $4,\left\|S_{1} u_{n}-u_{n}\right\| \rightarrow 0$ as $n \rightarrow \infty$. Hence, we get

$$
\lim _{n \rightarrow \infty}\left\|x_{n}-y_{n, 1}\right\|=0
$$

This implies that $\omega_{w}\left(x_{n}\right)=\omega_{w}\left(y_{n, 1}\right)$ and $\omega_{s}\left(x_{n}\right)=\omega_{s}\left(y_{n, 1}\right)$.

Take a point $q \in \omega_{w}\left(x_{n}\right)$ arbitrarily. Since $q \in \omega_{w}\left(u_{n}\right)$, by Step 4 and the demiclosedness principle, we have $q \in \operatorname{Fix}\left(S_{i}\right)$ for all $i \in\{1, \ldots, N\}$, that is, $q \in \bigcap_{i} \operatorname{Fix}\left(S_{i}\right)$. Moreover, note that

$$
\left\|y_{n, N}-x_{n}\right\| \leq \sum_{k=2}^{N}\left\|y_{n, k}-y_{n, k-1}\right\|+\left\|y_{n, 1}-x_{n}\right\|=\sum_{k=2}^{N} \beta_{n, k}\left\|S_{k} u_{n}-y_{n, k-1}\right\|+\left\|y_{n, 1}-x_{n}\right\|,
$$

and hence,

$$
\begin{aligned}
& \left\|x_{n}-T x_{n}\right\| \\
& \quad \leq\left\|x_{n}-x_{n+1}\right\|+\left\|x_{n+1}-T y_{n, N}\right\|+\left\|T y_{n, N}-T x_{n}\right\|
\end{aligned}
$$




$$
\begin{aligned}
\leq & \left\|x_{n}-x_{n+1}\right\|+\left\|\lambda_{n} \gamma\left(\alpha_{n} f\left(x_{n}\right)+\left(1-\alpha_{n}\right) S x_{n}\right)+\left(I-\lambda_{n} \mu F\right) T y_{n, N}-T y_{n, N}\right\| \\
& +\left\|y_{n, N}-x_{n}\right\| \\
= & \left\|x_{n}-x_{n+1}\right\|+\lambda_{n}\left\|\alpha_{n}\left(\gamma f\left(x_{n}\right)-\mu F T y_{n, N}\right)+\left(1-\alpha_{n}\right)\left(\gamma S x_{n}-\mu F T y_{n, N}\right)\right\| \\
& +\left\|y_{n, N}-x_{n}\right\| \\
\leq & \left\|x_{n}-x_{n+1}\right\|+\lambda_{n}\left[\left\|\gamma f\left(x_{n}\right)-\mu F T y_{n, N}\right\|+\left\|\gamma S x_{n}-\mu F T y_{n, N}\right\|\right]+\left\|y_{n, N}-x_{n}\right\| \\
\leq & \left\|x_{n}-x_{n+1}\right\|+\lambda_{n}\left[\left\|\gamma f\left(x_{n}\right)-\mu F T y_{n, N}\right\|+\left\|\gamma S x_{n}-\mu F T y_{n, N}\right\|\right] \\
& +\sum_{k=2}^{N} \beta_{n, k}\left\|S_{k} u_{n}-y_{n, k-1}\right\|+\left\|y_{n, 1}-x_{n}\right\| .
\end{aligned}
$$

So, it is easy to see that $\lim _{n \rightarrow \infty}\left\|y_{n, N}-x_{n}\right\|=0$ and $\lim _{n \rightarrow \infty}\left\|x_{n}-T x_{n}\right\|=0$ since $\| x_{n+1}-$ $x_{n}\left\|\rightarrow 0, \lambda_{n} \rightarrow 0,\right\| y_{n, 1}-x_{n} \| \rightarrow 0, \beta_{n, k} \rightarrow \beta_{k}$ and $\left\|S_{k} u_{n}-y_{n, k-1}\right\| \rightarrow 0$ for all $k \in\{1, \ldots, N\}$. Thus, by the demiclosedness principle, we have $q \in \operatorname{Fix}(T)$. Since $\left\{x_{n}\right\}$ is bounded and $\lim _{n \rightarrow \infty}\left\|x_{n}-T_{r_{n}} x_{n}\right\|=0$ (due to Step 3), by Lemma 2.3, we derive $q \in E P(\Theta, h)$. This shows that $q \in \Omega$. Therefore, we obtain the claim.

Step 6. $\left\{x_{n}\right\}$ converges strongly to a unique solution $x^{*}$ of Problem 1.2.

Indeed, according to $\left\|x_{n+1}-x_{n}\right\| \rightarrow 0$, we can take a subsequence $\left\{x_{n_{j}}\right\}$ of $\left\{x_{n}\right\}$ satisfying

$$
\begin{aligned}
\limsup _{n \rightarrow \infty}\left\langle(\gamma f-\mu F) x^{*}, x_{n+1}-x^{*}\right\rangle & =\limsup _{n \rightarrow \infty}\left\langle(\gamma f-\mu F) x^{*}, x_{n}-x^{*}\right\rangle \\
& =\lim _{j \rightarrow \infty}\left\langle(\gamma f-\mu F) x^{*}, x_{n_{j}}-x^{*}\right\rangle .
\end{aligned}
$$

Without loss of generality, we may further assume that $x_{n_{j}} \rightarrow \tilde{x}$; then $\tilde{x} \in \Omega$ as we just proved. Since $x^{*}$ is a solution of Problem 1.2, we get

$$
\limsup _{n \rightarrow \infty}\left\langle(\gamma f-\mu F) x^{*}, x_{n+1}-x^{*}\right\rangle=\left\langle(\gamma f-\mu F) x^{*}, \tilde{x}-x^{*}\right\rangle \leq 0 .
$$

Repeating the same argument as that of (3.10), we have

$$
\limsup _{n \rightarrow \infty}\left\langle(\gamma S-\mu F) x^{*}, x_{n+1}-x^{*}\right\rangle \leq 0
$$

From (1.13) and (3.1), it follows that (noticing that $x_{n+1}=P_{C} z_{n}$ and $0<\gamma \leq \tau$ )

$$
\begin{aligned}
& \left\|x_{n+1}-x^{*}\right\|^{2} \\
& =\left\langle z_{n}-x^{*}, x_{n+1}-x^{*}\right\rangle+\left\langle P_{C} z_{n}-z_{n}, P_{C} z_{n}-x^{*}\right\rangle \\
& \leq\left\langle z_{n}-x^{*}, x_{n+1}-x^{*}\right\rangle \\
& =\left\langle\left(I-\lambda_{n} \mu F\right) T y_{n, N}-\left(I-\lambda_{n} \mu F\right) x^{*}, x_{n+1}-x^{*}\right\rangle \\
& \quad+\alpha_{n} \lambda_{n} \gamma\left\langle f\left(x_{n}\right)-f\left(x^{*}\right), x_{n+1}-x^{*}\right\rangle+\lambda_{n}\left(1-\alpha_{n}\right) \gamma\left\langle S x_{n}-S x^{*}, x_{n+1}-x^{*}\right\rangle \\
& \quad+\alpha_{n} \lambda_{n}\left\langle(\gamma f-\mu F) x^{*}, x_{n+1}-x^{*}\right\rangle+\lambda_{n}\left(1-\alpha_{n}\right)\left\langle(\gamma S-\mu F) x^{*}, x_{n+1}-x^{*}\right\rangle \\
& \leq \\
& \quad\left[1-\lambda_{n} \tau+\alpha_{n} \lambda_{n} \gamma \rho+\lambda_{n}\left(1-\alpha_{n}\right) \gamma\right]\left\|x_{n}-x^{*}\right\|\left\|x_{n+1}-x^{*}\right\| \\
& \quad+\alpha_{n} \lambda_{n}\left\langle(\gamma f-\mu F) x^{*}, x_{n+1}-x^{*}\right\rangle+\lambda_{n}\left(1-\alpha_{n}\right)\left\langle(\gamma S-\mu F) x^{*}, x_{n+1}-x^{*}\right\rangle
\end{aligned}
$$




$$
\begin{aligned}
\leq & {\left[1-\alpha_{n} \lambda_{n} \gamma(1-\rho)\right]\left\|x_{n}-x^{*}\right\|\left\|x_{n+1}-x^{*}\right\| } \\
& +\alpha_{n} \lambda_{n}\left\langle(\gamma f-\mu F) x^{\prime \prime}, x_{n+1}-x^{\prime \prime}\right\rangle+\lambda_{n}\left(1-\alpha_{n}\right)\left\langle(\gamma S-\mu F) x^{* \prime}, x_{n+1}-x^{\prime \prime}\right\rangle \\
\leq & {\left[1-\alpha_{n} \lambda_{n} \gamma(1-\rho)\right] \frac{1}{2}\left(\left\|x_{n}-x^{\prime \prime}\right\|^{2}+\left\|x_{n+1}-x^{\prime \prime}\right\|^{2}\right) } \\
& +\alpha_{n} \lambda_{n}\left\langle(\gamma f-\mu F) x^{\prime \prime}, x_{n+1}-x^{\prime \prime}\right\rangle+\lambda_{n}\left(1-\alpha_{n}\right)\left\langle(\gamma S-\mu F) x^{\prime \prime}, x_{n+1}-x^{\prime \prime}\right\rangle .
\end{aligned}
$$

It turns out that

$$
\begin{aligned}
\| x_{n+1} & -x^{*} \|^{2} \\
\leq & \frac{1-\alpha_{n} \lambda_{n} \gamma(1-\rho)}{1+\alpha_{n} \lambda_{n} \gamma(1-\rho)}\left\|x_{n}-x^{*}\right\|^{2}+\frac{2}{1+\alpha_{n} \lambda_{n} \gamma(1-\rho)}\left[\alpha_{n} \lambda_{n}\left\langle(\gamma f-\mu F) x^{*}, x_{n+1}-x^{*}\right\rangle\right. \\
& \left.+\lambda_{n}\left(1-\alpha_{n}\right)\left\langle(\gamma S-\mu F) x^{*}, x_{n+1}-x^{*}\right\rangle\right] \\
\leq & {\left[1-\alpha_{n} \lambda_{n} \gamma(1-\rho)\right]\left\|x_{n}-x^{*}\right\|^{2} } \\
& +\frac{2}{1+\alpha_{n} \lambda_{n} \gamma(1-\rho)}\left[\alpha_{n} \lambda_{n}\left|(\gamma f-\mu F) x^{*}, x_{n+1}-x^{*}\right\rangle\right. \\
& \left.+\lambda_{n}\left(1-\alpha_{n}\right)\left\langle(\gamma S-\mu F) x^{*}, x_{n+1}-x^{*}\right\rangle\right] \\
= & {\left[1-\alpha_{n} \lambda_{n} \gamma(1-\rho)\right]\left\|x_{n}-x^{*}\right\|^{2}+\alpha_{n} \lambda_{n} \gamma(1-\rho)\left\{\frac{2}{\gamma(1-\rho)\left[1+\alpha_{n} \lambda_{n} \gamma(1-\rho)\right]}\right.} \\
& \times\left\langle(\gamma f-\mu F) x^{*}, x_{n+1}-x^{*}\right\rangle \\
& \left.+\frac{2\left(1-\alpha_{n}\right)}{\alpha_{n} \gamma(1-\rho)\left[1+\alpha_{n} \lambda_{n} \gamma(1-\rho)\right]}\left\langle(\gamma S-\mu F) x^{*}, x_{n+1}-x^{*}\right\rangle\right\} .
\end{aligned}
$$

Put $s_{n}=\left\|x_{n}-x^{*}\right\|^{2}, \gamma_{n}=\alpha_{n} \lambda_{n} \gamma(1-\rho)$ and

$$
\begin{aligned}
\delta_{n}= & \frac{2}{\gamma(1-\rho)\left[1+\alpha_{n} \lambda_{n} \gamma(1-\rho)\right]}\left\langle(\gamma f-\mu F) x^{*}, x_{n+1}-x^{*}\right\rangle \\
& +\frac{2\left(1-\alpha_{n}\right)}{\alpha_{n} \gamma(1-\rho)\left[1+\alpha_{n} \lambda_{n} \gamma(1-\rho)\right]}\left\langle(\gamma S-\mu F) x^{*}, x_{n+1}-x^{*}\right\rangle .
\end{aligned}
$$

Then (3.12) can be rewritten as

$$
s_{n+1} \leq\left(1-\gamma_{n}\right) s_{n}+\gamma_{n} \delta_{n} .
$$

From conditions $(\mathrm{C} 1)$ and $(\mathrm{C} 2)$, we conclude from $0<1-\rho \leq 1$ that

$$
\left\{\gamma_{n}\right\} \subset[0,1] \text { and } \sum_{n=1}^{\infty} \gamma_{n}=\infty
$$

Note that

$$
\frac{2}{\gamma(1-\rho)\left[1+\alpha_{n} \lambda_{n} \gamma(1-\rho)\right]} \leq \frac{2}{\gamma(1-\rho)}
$$

and

$$
\frac{2\left(1-\alpha_{n}\right)}{\alpha_{n} \gamma(1-\rho)\left[1+\alpha_{n} \lambda_{n} \gamma(1-\rho)\right]} \leq \frac{2}{a \gamma(1-\rho)} .
$$


Consequently, utilizing Lemma 2.1, we obtain that

$$
\begin{aligned}
\limsup _{n \rightarrow \infty} \delta_{n} \leq & \limsup _{n \rightarrow \infty} \frac{2}{\gamma(1-\rho)\left[1+\alpha_{n} \lambda_{n} \gamma(1-\rho)\right]}\left\langle(\gamma f-\mu F) x^{*}, x_{n+1}-x^{*}\right\rangle \\
& +\limsup _{n \rightarrow \infty} \frac{2\left(1-\alpha_{n}\right)}{\alpha_{n} \gamma(1-\rho)\left[1+\alpha_{n} \lambda_{n} \gamma(1-\rho)\right]}\left\langle(\gamma S-\mu F) x^{*}, x_{n+1}-x^{*}\right\rangle \\
\leq & 0 .
\end{aligned}
$$

So, this together with Lemma 2.1 leads to $\lim _{n \rightarrow \infty}\left\|x_{n}-x^{*}\right\|=0$. The proof is complete.

We now derive the following strong convergence result for a sequence generated by Algorithm 1.1 to a unique solution of Problem 1.3.

Theorem 3.2 Let $\left\{\lambda_{n}\right\},\left\{\alpha_{n}\right\},\left\{\beta_{n, i}\right\}, i=1, \ldots, N$ be sequences in $(0,1)$ such that $\beta_{n, i} \rightarrow \beta_{i} \in$ $(0,1)$ as $n \rightarrow \infty$ for all $i \in\{1, \ldots, N\}$. Assume that the solution set $\Xi$ of HVIP (1.16) is nonempty and that the following conditions hold:

(C1) $0<\liminf _{n \rightarrow \infty} \alpha_{n} \leq \lim \sup _{n \rightarrow \infty} \alpha_{n}<1$;

(C2) $\lim _{n \rightarrow \infty} \lambda_{n}=0$ and $\sum_{n=1}^{\infty} \lambda_{n}=\infty$;

(C3) $\sum_{n=2}^{\infty}\left|\alpha_{n} \lambda_{n}-\alpha_{n-1} \lambda_{n-1}\right|<\infty$ or $\lim _{n \rightarrow \infty} \frac{\left|\alpha_{n} \lambda_{n}-\alpha_{n-1} \lambda_{n-1}\right|}{\lambda_{n}}=0$;

(C4) $\sum_{n=2}^{\infty}\left|\lambda_{n}-\lambda_{n-1}\right|<\infty$ or $\lim _{n \rightarrow \infty} \frac{\left|\lambda_{n}-\lambda_{n-1}\right|}{\lambda_{n}}=0$;

(C5) $\sum_{n=2}^{\infty}\left|\beta_{n, i}-\beta_{n-1, i}\right|<\infty$ or $\lim _{n \rightarrow \infty} \frac{\left|\beta_{n, i}-\beta_{n-1, i}\right|}{\lambda_{n}}=0$ for all $i \in\{1, \ldots, N\}$;

(C6) $\sum_{n=2}^{\infty}\left|r_{n}-r_{n-1}\right|<\infty$ or $\lim _{n \rightarrow \infty} \frac{\left|r_{n}-r_{n-1}\right|}{\lambda_{n}}=0$;

(C7) there are constants $\bar{k}, \theta>0$ satisfying $\|x-T x\| \geq \bar{k}[d(x, \Omega)]^{\theta}$ for all $x \in C$.

Then the following assertions hold.

(a) Let $\left\{x_{n}\right\}$ be a sequence generated by the scheme (1.13), then $\left\{x_{n}\right\}$ converges strongly to a unique solution $x^{*} \in \Omega$ of Problem 1.3 provided $\omega_{w}\left(x_{n}\right) \subset \Xi$.

(b) Let $\left\{x_{n}\right\}$ be a sequence generated by the scheme (1.14), then $\left\{x_{n}\right\}$ converges strongly to a unique solution $x^{*}$ of the following VIP provided $\omega_{w}\left(x_{n}\right) \subset \Xi$ :

$$
\text { find } x^{*} \in \Xi \text { such that }\left\langle F x^{*}, x-x^{*}\right\rangle \geq 0, \quad \forall x \in \Xi \text {. }
$$

Proof For part (a), suppose that the sequence $\left\{x_{n}\right\}$ is generated by the scheme (1.13). First of all, from the condition $\Xi \neq \emptyset$ it follows that $\Omega=\operatorname{Fix}(T) \cap\left(\bigcap_{i} \operatorname{Fix}\left(S_{i}\right)\right) \cap E P(\Theta, h) \neq \emptyset$. Note that $0<\gamma \leq \tau$ and $\kappa \geq \eta \Leftrightarrow \mu \eta \geq \tau$. Hence, it follows from the $\rho$-contractiveness of $f$ and $\gamma \rho<\gamma \leq \tau \leq \mu \eta$ that $\mu F-\gamma f$ is $(\mu \eta-\gamma \rho)$-strongly monotone and Lipschitz continuous. So, there exists a unique solution $x^{*}$ of the following VIP:

$$
\text { find } x^{*} \in \Xi \text { such that }\left\langle(\mu F-\gamma f) x^{*}, x-x^{*}\right\rangle \geq 0, \quad \forall x \in \Xi \text {. }
$$

Consequently, it is easy to see that Problem 1.3 has a unique solution $x^{\prime \prime} \in \Xi$. In addition, taking into account condition (C1), without loss of generality, we may assume that $\left\{\alpha_{n}\right\} \subset$ $[a, b]$ for some $a, b \in(0,1)$.

The rest of the proof is divided into several steps.

Step 1 . The sequences $\left\{x_{n}\right\},\left\{y_{n, i}\right\}$ for all $i,\left\{u_{n}\right\}$ are bounded.

Indeed, repeating the same argument as in Step 1 of the proof of Theorem 3.1, we can derive the claim. 
Step $2 . \lim _{n \rightarrow \infty}\left\|x_{n+1}-x_{n}\right\|=0$, that is, $\left\{x_{n}\right\}$ is asymptotically regular.

Indeed, repeating the same argument as in Step 2 of the proof of Theorem 3.1, we can derive the claim.

Step 3. $\left\|x_{n}-u_{n}\right\|=\left\|x_{n}-T_{r_{n}} x_{n}\right\| \rightarrow 0$ as $n \rightarrow \infty$.

Indeed, repeating the same argument as in Step 3 of the proof of Theorem 3.1, we can derive the claim.

Step 4. For all $i \in\{1, \ldots, N\},\left\|S_{i} u_{n}-u_{n}\right\| \rightarrow 0$ as $n \rightarrow \infty$.

Indeed, repeating the same argument as in Step 4 of the proof of Theorem 3.1, we can derive the claim.

Step 5. $\lim _{n \rightarrow \infty}\left\|y_{n, N}-x_{n}\right\|=\lim _{n \rightarrow \infty}\left\|x_{n}-T x_{n}\right\|=0$ and $\omega_{w}\left(x_{n}\right) \subset \Omega$.

Indeed, repeating the same argument as in Step 5 of the proof of Theorem 3.1, we can derive the claim.

Step 6. $\left\{x_{n}\right\}$ converges strongly to a unique solution $x^{*}$ of Problem 1.3.

Indeed, we now take a subsequence $\left\{x_{n_{j}}\right\}$ of $\left\{x_{n}\right\}$ satisfying

$$
\limsup _{n \rightarrow \infty}\left\langle(\mu F-\gamma f) x^{*}, x_{n}-x^{*}\right\rangle=\lim _{j \rightarrow \infty}\left\langle(\mu F-\gamma f) x^{*}, x_{n_{j}}-x^{*}\right\rangle .
$$

Without loss of generality, we may further assume that $x_{n_{j}} \rightarrow \tilde{x}$; then $\tilde{x} \in \Xi$ according to the assumption $\omega_{w}\left(x_{n}\right) \subset \Xi$. Since $x^{*}$ is a solution of THVIP (1.15), we get

$$
\limsup _{n \rightarrow \infty}\left\langle(\mu F-\gamma f) x^{*}, x_{n}-x^{*}\right\rangle=\left\langle(\mu F-\gamma f) x^{*}, \tilde{x}-x^{*}\right\rangle \geq 0 \text {. }
$$

From (1.13) and (3.1), it follows that (noticing that $x_{n+1}=P_{C} z_{n}$ and $0<\gamma \leq \tau$ )

$$
\begin{aligned}
\left\|x_{n+1}-x^{*}\right\|^{2} & \\
= & \left\langle z_{n}-x^{*}, x_{n+1}-x^{*}\right\rangle+\left\langle P_{C} z_{n}-z_{n}, P_{C} z_{n}-x^{*}\right\rangle \\
\leq & \left\langle z_{n}-x^{*}, x_{n+1}-x^{*}\right\rangle \\
= & \left\langle\left(I-\lambda_{n} \mu F\right) T y_{n, N}-\left(I-\lambda_{n} \mu F\right) x^{*}, x_{n+1}-x^{*}\right\rangle \\
& +\alpha_{n} \lambda_{n} \gamma\left\langle f\left(x_{n}\right)-f\left(x^{*}\right), x_{n+1}-x^{*}\right\rangle+\lambda_{n}\left(1-\alpha_{n}\right) \gamma\left\langle S x_{n}-S x^{*}, x_{n+1}-x^{*}\right\rangle \\
& +\alpha_{n} \lambda_{n}\left\langle(\gamma f-\mu F) x^{*}, x_{n+1}-x^{*}\right\rangle+\lambda_{n}\left(1-\alpha_{n}\right)\left\langle(\gamma S-\mu F) x^{*}, x_{n+1}-x^{*}\right\rangle \\
\leq & {\left[1-\lambda_{n} \tau+\alpha_{n} \lambda_{n} \gamma \rho+\lambda_{n}\left(1-\alpha_{n}\right) \gamma\right]\left\|x_{n}-x^{*}\right\|\left\|x_{n+1}-x^{* *}\right\| } \\
& +\alpha_{n} \lambda_{n}\left\langle(\gamma f-\mu F) x^{*}, x_{n+1}-x^{*}\right\rangle+\lambda_{n}\left(1-\alpha_{n}\right)\left\langle(\gamma S-\mu F) x^{*}, x_{n+1}-x^{*}\right\rangle \\
\leq & {\left[1-\alpha_{n} \lambda_{n} \gamma(1-\rho)\right]\left\|x_{n}-x^{*}\right\|\left\|x_{n+1}-x^{*}\right\| } \\
& +\alpha_{n} \lambda_{n}\left\langle(\gamma f-\mu F) x^{*}, x_{n+1}-x^{*}\right\rangle+\lambda_{n}\left(1-\alpha_{n}\right)\left\langle(\gamma S-\mu F) x^{*}, x_{n+1}-x^{*}\right\rangle \\
\leq & {\left[1-\alpha_{n} \lambda_{n} \gamma(1-\rho)\right] \frac{1}{2}\left(\left\|x_{n}-x^{*}\right\|^{2}+\left\|x_{n+1}-x^{*}\right\|^{2}\right) } \\
& +\alpha_{n} \lambda_{n}\left\langle(\gamma f-\mu F) x^{*}, x_{n+1}-x^{*}\right\rangle+\lambda_{n}\left(1-\alpha_{n}\right)\left\langle(\gamma S-\mu F) x^{*}, x_{n+1}-x^{*}\right\rangle .
\end{aligned}
$$

It turns out that

$$
\begin{aligned}
& \left\|x_{n+1}-x^{*}\right\|^{2} \\
& \quad \leq \frac{1-\alpha_{n} \lambda_{n} \gamma(1-\rho)}{1+\alpha_{n} \lambda_{n} \gamma(1-\rho)}\left\|x_{n}-x^{*}\right\|^{2}+\frac{2}{1+\alpha_{n} \lambda_{n} \gamma(1-\rho)}\left[\alpha_{n} \lambda_{n}\left\langle(\gamma f-\mu F) x^{*}, x_{n+1}-x^{*}\right\rangle\right.
\end{aligned}
$$




$$
\begin{aligned}
& \left.+\lambda_{n}\left(1-\alpha_{n}\right)\left\langle(\gamma S-\mu F) x^{*}, x_{n+1}-x^{*}\right\rangle\right] \\
\leq & {\left[1-\alpha_{n} \lambda_{n} \gamma(1-\rho)\right]\left\|x_{n}-x^{*}\right\|^{2}+\frac{2}{1+\alpha_{n} \lambda_{n} \gamma(1-\rho)}\left[\alpha_{n} \lambda_{n}\left\langle(\gamma f-\mu F) x^{*}, x_{n+1}-x^{*}\right\rangle\right.} \\
& \left.+\lambda_{n}\left(1-\alpha_{n}\right)\left\langle(\gamma S-\mu F) x^{*}, x_{n+1}-x^{*}\right\rangle\right] .
\end{aligned}
$$

However, from $x^{*} \in \Xi$ and condition (C7), we obtain

$$
\begin{aligned}
& \left\langle(\gamma S-\mu F) x^{*}, x_{n+1}-x^{*}\right\rangle \\
& \quad=\left\langle(\gamma S-\mu F) x^{*}, x_{n+1}-P_{\Omega} x_{n+1}\right\rangle+\left\langle(\gamma S-\mu F) x^{*}, P_{\Omega} x_{n+1}-x^{*}\right\rangle \\
& \quad \leq\left\langle(\gamma S-\mu F) x^{*}, x_{n+1}-P_{\Omega} x_{n+1}\right\rangle \\
& \leq\left\|(\gamma S-\mu F) x^{*}\right\| d\left(x_{n+1}, \Omega\right) \\
& \quad \leq\left\|(\gamma S-\mu F) x^{*}\right\|\left(\frac{1}{\bar{k}}\left\|x_{n+1}-T x_{n+1}\right\|\right)^{1 / \theta} .
\end{aligned}
$$

On the other hand, we also have

$$
\begin{aligned}
& \left\|x_{n+1}-T x_{n+1}\right\| \\
& \quad \leq\left\|x_{n+1}-T x_{n}\right\|+\left\|T x_{n}-T x_{n+1}\right\| \\
& \quad \leq\left\|x_{n}-x_{n+1}\right\|+\left\|\lambda_{n} \gamma\left(\alpha_{n} f\left(x_{n}\right)+\left(1-\alpha_{n}\right) S x_{n}\right)+\left(I-\lambda_{n} \mu F\right) T y_{n, N}-T x_{n}\right\| \\
& \quad \leq\left\|x_{n}-x_{n+1}\right\|+\left\|T y_{n, N}-T x_{n}\right\|+\lambda_{n}\left\|\gamma\left(\alpha_{n} f\left(x_{n}\right)+\left(1-\alpha_{n}\right) S x_{n}\right)-\mu F T y_{n, N}\right\| \\
& \quad=\left\|x_{n}-x_{n+1}\right\|+\left\|T y_{n, N}-T x_{n}\right\|+\lambda_{n}\left\|\gamma \alpha_{n}\left(f\left(x_{n}\right)-S x_{n}\right)+\gamma S x_{n}-\mu F T y_{n, N}\right\| \\
& \quad \leq\left\|x_{n}-x_{n+1}\right\|+\left\|y_{n, N}-x_{n}\right\|+\lambda_{n} M_{0} .
\end{aligned}
$$

Hence, for a big enough constant $\bar{k}_{1}>0$, we have

$$
\left\langle(\gamma S-\mu F) x^{\prime \prime}, x_{n+1}-x^{\prime \prime}\right\rangle \leq \bar{k}_{1}\left(\left\|x_{n}-x_{n+1}\right\|+\left\|y_{n, N}-x_{n}\right\|+\lambda_{n} M_{0}\right)^{1 / \theta} .
$$

Combining (3.14)-(3.17), we get

$$
\begin{aligned}
\left\|x_{n+1}-x^{*}\right\|^{2} & \\
\leq & {\left[1-\alpha_{n} \lambda_{n} \gamma(1-\rho)\right]\left\|x_{n}-x^{*}\right\|^{2}+\frac{2}{1+\alpha_{n} \lambda_{n} \gamma(1-\rho)}\left[\alpha_{n} \lambda_{n}\left\langle(\gamma f-\mu F) x^{*}, x_{n+1}-x^{*}\right\rangle\right.} \\
& \left.+\lambda_{n}\left(1-\alpha_{n}\right)\left\langle(\gamma S-\mu F) x^{*}, x_{n+1}-x^{*}\right\rangle\right] \\
= & {\left[1-\alpha_{n} \lambda_{n} \gamma(1-\rho)\right]\left\|x_{n}-x^{*}\right\|^{2} } \\
& +\alpha_{n} \lambda_{n} \gamma(1-\rho)\left[\frac{2}{\gamma(1-\rho)\left[1+\alpha_{n} \lambda_{n} \gamma(1-\rho)\right]}\left\langle(\gamma f-\mu F) x^{*}, x_{n+1}-x^{*}\right\rangle\right. \\
& \left.+\frac{2\left(1-\alpha_{n}\right)}{\alpha_{n} \gamma(1-\rho)\left[1+\alpha_{n} \lambda_{n} \gamma(1-\rho)\right]}\left\langle(\gamma S-\mu F) x^{*}, x_{n+1}-x^{*}\right\rangle\right] \\
\leq & {\left[1-\alpha_{n} \lambda_{n} \gamma(1-\rho)\right]\left\|x_{n}-x^{*}\right\|^{2} } \\
& +\alpha_{n} \lambda_{n} \gamma(1-\rho)\left[\frac{2}{\gamma(1-\rho)\left[1+\alpha_{n} \lambda_{n} \gamma(1-\rho)\right]}\left\langle(\gamma f-\mu F) x^{*}, x_{n+1}-x^{*}\right\rangle\right.
\end{aligned}
$$




$$
\begin{aligned}
& \left.\quad+\frac{2\left(1-\alpha_{n}\right)}{\alpha_{n} \gamma(1-\rho)\left[1+\alpha_{n} \lambda_{n} \gamma(1-\rho)\right]} \bar{k}_{1}\left(\left\|x_{n}-x_{n+1}\right\|+\left\|y_{n, N}-x_{n}\right\|+\lambda_{n} M_{0}\right)^{1 / \theta}\right] \\
& =\left(1-\gamma_{n}\right)\left\|x_{n}-x^{*}\right\|^{2}+\gamma_{n} \delta_{n},
\end{aligned}
$$

where $\gamma_{n}=\alpha_{n} \lambda_{n} \gamma(1-\rho)$ and

$$
\begin{aligned}
\delta_{n}= & \frac{2}{\gamma(1-\rho)\left[1+\alpha_{n} \lambda_{n} \gamma(1-\rho)\right]}\left\langle(\gamma f-\mu F) x^{*}, x_{n+1}-x^{*}\right\rangle \\
& +\frac{2\left(1-\alpha_{n}\right)}{\alpha_{n} \gamma(1-\rho)\left[1+\alpha_{n} \lambda_{n} \gamma(1-\rho)\right]} \bar{k}_{1}\left(\left\|x_{n}-x_{n+1}\right\|+\left\|y_{n, N}-x_{n}\right\|+\lambda_{n} M_{0}\right)^{1 / \theta} .
\end{aligned}
$$

Now, conditions (C1) and (C2) imply that $\sum_{n=1}^{\infty} \gamma_{n}=\infty$. Moreover, since $\left\|x_{n+1}-x_{n}\right\| \rightarrow 0$ (due to Step 2), $\left\|y_{n, N}-x_{n}\right\| \rightarrow 0$ (due to Step 5) and $\lambda_{n} \rightarrow 0$, we obtain from (3.13) that

$$
\begin{aligned}
\delta_{n}= & \frac{2}{\gamma(1-\rho)\left[1+\alpha_{n} \lambda_{n} \gamma(1-\rho)\right]}\left[\left\langle(\gamma f-\mu F) x^{*}, x_{n+1}-x_{n}\right\rangle+\left\langle(\gamma f-\mu F) x^{*}, x_{n}-x^{*}\right\rangle\right] \\
& +\frac{2\left(1-\alpha_{n}\right)}{\alpha_{n} \gamma(1-\rho)\left[1+\alpha_{n} \lambda_{n} \gamma(1-\rho)\right]} \bar{k}_{1}\left(\left\|x_{n}-x_{n+1}\right\|+\left\|y_{n, N}-x_{n}\right\|+\lambda_{n} M_{0}\right)^{1 / \theta} \\
\leq & \frac{2}{\gamma(1-\rho)\left[1+\alpha_{n} \lambda_{n} \gamma(1-\rho)\right]}\left[\left\|(\gamma f-\mu F) x^{*}\right\|\left\|x_{n+1}-x_{n}\right\|+\left\langle(\gamma f-\mu F) x^{*}, x_{n}-x^{*}\right\rangle\right] \\
& +\frac{2(1-a)}{a \gamma(1-\rho)\left[1+\alpha_{n} \lambda_{n} \gamma(1-\rho)\right]} \bar{k}_{1}\left(\left\|x_{n}-x_{n+1}\right\|+\left\|y_{n, N}-x_{n}\right\|+\lambda_{n} M_{0}\right)^{1 / \theta},
\end{aligned}
$$

which together with Lemma 2.4 leads to

$$
\limsup _{n \rightarrow \infty} \delta_{n} \leq 0
$$

Therefore, we can apply Lemma 2.1 to (3.18) to conclude that $x_{n} \rightarrow x^{*}$. The proof of part (a) is complete. It is easy to see that part (b) now becomes a straightforward consequence of part (a) since, if $f=0$, THVIP (1.15) reduces to the VIP in part (b). This completes the proof.

Utilizing Theorem 3.2, we immediately derive the following result.

Corollary 3.1 Let $F: C \rightarrow H$ be a $\kappa$-Lipschitzian and $\eta$-strongly monotone operator with constants $\kappa, \eta>0$, respectively, $f: C \rightarrow H$ be a $\rho$-contraction with a coefficient $\rho \in[0,1)$ and $S, T: C \rightarrow C$ be nonexpansive mappings with $\operatorname{Fix}(T) \neq \emptyset$. Let $0<\mu<2 \eta / \kappa^{2}$ and $0<$ $\gamma \leq \tau$, where $\tau=1-\sqrt{1-\mu\left(2 \eta-\mu \kappa^{2}\right)}$. Assume that the solution set $\Xi$ of HVIP (1.11) is nonempty and the following conditions hold for two sequences $\left\{\lambda_{n}\right\},\left\{\alpha_{n}\right\} \subset(0,1)$ :

(i) $0<\liminf _{n \rightarrow \infty} \alpha_{n} \leq \limsup _{n \rightarrow \infty} \alpha_{n}<1$;

(ii) $\lim _{n \rightarrow \infty} \lambda_{n}=0$ and $\sum_{n=1}^{\infty} \lambda_{n}=\infty$;

(iii) $\sum_{n=2}^{\infty}\left|\alpha_{n} \lambda_{n}-\alpha_{n-1} \lambda_{n-1}\right|<\infty$ or $\lim _{n \rightarrow \infty} \frac{\left|\alpha_{n} \lambda_{n}-\alpha_{n-1} \lambda_{n-1}\right|}{\lambda_{n}}=0$;

(iv) $\sum_{n=2}^{\infty}\left|\lambda_{n}-\lambda_{n-1}\right|<\infty$ or $\lim _{n \rightarrow \infty} \frac{\left|\lambda_{n}-\lambda_{n-1}\right|}{\lambda_{n}}=0$;

(v) there are constants $\bar{k}, \theta>0$ satisfying $\|x-T x\| \geq \bar{k}[d(x, \Omega)]^{\theta}$ for all $x \in C$.

Then the following assertions hold. 
(a) Let $\left\{x_{n}\right\}$ be a sequence generated from an arbitrary initial $x_{1} \in C$ by the iterative scheme

$$
x_{n+1}=P_{C}\left[\lambda_{n} \gamma\left(\alpha_{n} f\left(x_{n}\right)+\left(1-\alpha_{n}\right) S x_{n}\right)+\left(I-\lambda_{n} \mu F\right) T x_{n}\right], \quad n \geq 1,
$$

such that $\omega_{w}\left(x_{n}\right) \subset \Xi$, then $\left\{x_{n}\right\}$ converges in norm to the point $x^{*} \in \operatorname{Fix}(T)$ which is a unique solution of Problem 1.1.

(b) Let $\left\{x_{n}\right\}$ be a sequence generated from an arbitrary initial $x_{1} \in C$ by the iterative scheme

$$
x_{n+1}=P_{C}\left[\lambda_{n}\left(1-\alpha_{n}\right) \gamma S x_{n}+\left(I-\lambda_{n} \mu F\right) T x_{n}\right], \quad n \geq 1,
$$

such that $\omega_{w}\left(x_{n}\right) \subset \Xi$, then $\left\{x_{n}\right\}$ converges in norm to a unique solution $x^{*}$ of the VIP offinding $x^{*} \in \Xi$ such that

$$
\left\langle F x^{*}, x-x^{*}\right| \geq 0, \quad \forall x \in \Xi \text {. }
$$

Proof In Theorem 3.2, putting $\Theta=h=0, S_{i}=I, i=1, \ldots, N$, from (1.13) we obtain that $x_{n}=u_{n}=y_{n, i}, i=1, \ldots, N$. In this case, $\Omega=\operatorname{Fix}(T)$ and (1.13) reduces to (3.19). Moreover, it is easy to see that Problem 1.3 reduces to Problem 1.1. Thus, by Theorem 3.2 we obtain the desired results.

Remark 3.1 Corollary 3.1 improves and extends [29, Theorem 4.1] in the following aspects:

(a) The restriction $\lim _{n \rightarrow 0} \alpha_{n}=0$ in [29, Theorem 4.1] is replaced by

$$
0<\liminf _{n \rightarrow \infty} \alpha_{n} \leq \limsup _{n \rightarrow \infty} \alpha_{n}<1
$$

(b) The condition $\lim _{n \rightarrow 0} \lambda_{n}^{1 / \theta} / \alpha_{n}=0$ is not assumed in Corollary 3.1;

(c) The boundedness of the sequence $\left\{x_{n}\right\}$ is not assumed in Corollary 3.1.

Very recently, Yao et al. [18] considered the following HVIP of finding $x^{*} \in \operatorname{Fix}(T)$ such that

$$
\left\langle(I-S) x^{*}, x-x^{*}\right\rangle \geq 0, \quad \forall x \in \operatorname{Fix}(T)
$$

where $T, S: C \rightarrow C$ are two nonexpansive mappings and $\operatorname{Fix}(T)$ is the fixed point set of $T$. Let $\Omega$ denote the solution set of HVIP (3.20) and assume that $\Omega$ is nonempty; consequently, the metric projection $P_{\Omega}$ is well defined. It is interesting to find the minimumnorm solution $x^{*}$ of HVIP (3.20) which exists uniquely and is exactly the nearest point projection of the origin to $\Omega$, that is, $x^{*}=P_{\Omega}(0)$. Alternatively, $x^{*}$ is the unique solution of the quadratic minimization problem:

$$
\left\|x^{*}\right\|^{2}=\min \left\{\|x\|^{2}: x \in \Omega\right\}
$$

They used the contractions to regularize the nonexpansive mapping $S$ to introduce an explicit scheme that generates a sequence $\left\{x_{n}\right\}$ via an iterative algorithm and proved that this sequence converges strongly to the minimum-norm solution $x^{*}$ of HVIP (3.20). 
In Corollary 3.1, if we put $\mu=2, F=\frac{1}{2} I$ and $\gamma=\tau=1$, then HVIP (1.11) reduces to HVIP (3.20) and $\Xi=\Omega$. In this case, THVIP (1.10) reduces to the quadratic minimization problem (3.21). In terms of Corollary 3.1(a), $\left\{x_{n}\right\}$ converges in norm to the point $x^{*} \in \operatorname{Fix}(T)$ which is a unique solution of VIP (1.2); see (3.22). Also, by Corollary 3.1(b), $\left\{x_{n}\right\}$ converges in norm to the minimum-norm solution of HVIP (3.20). Therefore, we get the following conclusions.

Corollary 3.2 Letf $: C \rightarrow H$ be a $\rho$-contraction with a coefficient $\rho \in[0,1)$ and $S, T: C \rightarrow$ $C$ be two nonexpansive mappings with $\operatorname{Fix}(T) \neq \emptyset$. Assume that the solution set $\Omega$ of HVIP (3.20) is nonempty and that the following conditions hold for two sequences $\left\{\lambda_{n}\right\},\left\{\alpha_{n}\right\} \subset$ $(0,1)$ :

(i) $0<\liminf _{n \rightarrow \infty} \alpha_{n} \leq \limsup _{n \rightarrow \infty} \alpha_{n}<1$;

(ii) $\lim _{n \rightarrow \infty} \lambda_{n}=0$ and $\sum_{n=1}^{\infty} \lambda_{n}=\infty$;

(iii) $\sum_{n=2}^{\infty}\left|\alpha_{n} \lambda_{n}-\alpha_{n-1} \lambda_{n-1}\right|<\infty$ or $\lim _{n \rightarrow \infty} \frac{\left|\alpha_{n} \lambda_{n}-\alpha_{n-1} \lambda_{n-1}\right|}{\lambda_{n}}=0$;

(iv) $\sum_{n=2}^{\infty}\left|\lambda_{n}-\lambda_{n-1}\right|<\infty$ or $\lim _{n \rightarrow \infty} \frac{\left|\lambda_{n}-\lambda_{n-1}\right|}{\lambda_{n}}=0$;

(v) there are constants $\bar{k}, \theta>0$ satisfying $\|x-T x\| \geq \bar{k}[d(x, \Omega)]^{\theta}$ for all $x \in C$.

Then the following assertions hold.

(a) Let $\left\{x_{n}\right\}$ be a sequence generated from an arbitrary initial $x_{1} \in C$ by the iterative scheme

$$
x_{n+1}=P_{C}\left[\lambda_{n}\left(\alpha_{n} f\left(x_{n}\right)+\left(1-\alpha_{n}\right) S x_{n}\right)+\left(1-\lambda_{n}\right) T x_{n}\right], \quad n \geq 1,
$$

such that $\omega_{w}\left(x_{n}\right) \subset \Omega$, then $\left\{x_{n}\right\}$ converges in norm to the point $x^{*} \in \operatorname{Fix}(T)$ which is a unique solution of the VIP of finding $x^{*} \in \Omega$ such that

$$
\left\langle(I-f) x^{*}, x-x^{*}\right\rangle \geq 0, \quad \forall x \in \Omega \text {. }
$$

(b) Let $\left\{x_{n}\right\}$ be a sequence generated from an arbitrary initial $x_{1} \in C$ by the iterative scheme

$$
x_{n+1}=P_{C}\left[\lambda_{n}\left(1-\alpha_{n}\right) S x_{n}+\left(1-\lambda_{n}\right) T x_{n}\right], \quad n \geq 1,
$$

such that $\omega_{w}\left(x_{n}\right) \subset \Omega$, then $\left\{x_{n}\right\}$ converges in norm to a minimum-norm solution of HVIP (3.20).

\section{Applications}

Let $C$ be a nonempty, closed and convex subset of a real Hilbert space $H$. Recall that a point $u \in C$ is a solution to VIP (1.1) if and only if

$$
u=P_{C}(I-\lambda A) u, \quad \lambda>0 .
$$

Definition 4.1 An operator $A: C \rightarrow H$ is said to be an $\alpha$-inverse strongly monotone operator if there exists a constant $\alpha>0$ such that

$$
\langle A x-A y, x-y\rangle \geq \alpha\|A x-A y\|^{2}, \quad \forall x, y \in C .
$$


As an example, we recall that the $\alpha$-inverse strongly monotone operators are firmly nonexpansive mappings if $\alpha \geq 1$ and that every $\alpha$-inverse strongly monotone operator is also $\frac{1}{\alpha}$-Lipschitz continuous (see [37]).

Let us observe also that, if $A$ is $\alpha$-inverse strongly monotone, the mappings $P_{C}(I-\lambda A)$ are nonexpansive for all $\lambda \in(0,2 \alpha]$ since they are compositions of nonexpansive mappings (see [37, pp.419]).

Let us consider $\tilde{S}_{1}, \ldots, \tilde{S}_{M}$ to be a finite number of nonexpansive self-mappings on $C$ and $A_{1}, \ldots, A_{N}$ to be a finite number of $\alpha$-inverse strongly monotone operators. Let $T$ be a nonexpansive self-mapping on $C$. Very recently, Marino, Muglia and Yao [27] considered an application of Theorem 3.12 of [27], to solve the following mixed problem.

To find $x^{*} \in \operatorname{Fix}(T) \cap E P(\Theta, h)$ such that

$$
\left\{\begin{array}{l}
\left\langle\left(I-\tilde{S}_{1}\right) x^{*}, y-x^{*}\right\rangle \geq 0, \quad \forall y \in \operatorname{Fix}(T) \cap E P(\Theta, h), \\
\left\langle\left(I-\tilde{S}_{2}\right) x^{*}, y-x^{*}\right\rangle \geq 0, \quad \forall y \in \operatorname{Fix}(T) \cap E P(\Theta, h), \\
\cdots \\
\left\langle\left(I-\tilde{S}_{M}\right) x^{*}, y-x^{*}\right\rangle \geq 0, \quad \forall y \in \operatorname{Fix}(T) \cap E P(\Theta, h), \\
\left\langle A_{1} x^{*}, y-x^{*}\right\rangle \geq 0, \quad \forall y \in C, \\
\left\langle A_{2} x^{*}, y-x^{*}\right\rangle \geq 0, \quad \forall y \in C, \\
\cdots \\
\left\langle A_{N} x^{*}, y-x^{*}\right\rangle \geq 0, \quad \forall y \in C .
\end{array}\right.
$$

Let us call $(S V I P)$ the set of solutions of the $(N+M)$-system. This problem is equivalent to finding a common fixed point of $T,\left\{P_{\mathrm{Fix}(T) \cap E P(\Theta, h)} \tilde{S}_{i}\right\}_{i=1}^{M},\left\{P_{C}\left(I-\lambda A_{i}\right)\right\}_{i=1}^{N}$.

Based on the above mixed problem, in this section we first consider the following more general mixed problem.

Problem 4.1 Let $F: C \rightarrow H$ be $\kappa$-Lipschitzian and $\eta$-strongly monotone on $C, f: C \rightarrow H$ be a $\rho$-contraction with a coefficient $\rho \in[0,1)$ and $S, T$ be nonexpansive self-mappings on $C$. Let $0<\mu<2 \eta / \kappa^{2}$ and $0<\gamma \leq \tau$, where $\tau=1-\sqrt{1-\mu\left(2 \eta-\mu \kappa^{2}\right)}$. Assume that $\Theta, h: C \times C \rightarrow \mathbb{R}$ are two bifunctions. Then the objective is to find $x^{\prime \prime} \in \Omega$ such that

$$
\begin{cases}\left\langle(\mu F-\gamma f) x^{*}, x-x^{*}\right\rangle \geq 0, & \forall x \in \Omega, \\ \left\langle(\mu F-\gamma S) x^{*}, y-x^{*}\right\rangle \geq 0, & \forall y \in \Omega,\end{cases}
$$

where $\Omega=\operatorname{Fix}(T) \cap(S V I P) \cap E P(\Theta, h) \neq \emptyset$.

Utilizing Theorem 3.1 we obtain the following result.

Theorem 4.1 Assume that Problem 4.1 has a solution. Let $0<\lambda \leq 2 \alpha$. Let $\left\{\lambda_{n}\right\},\left\{\alpha_{n}\right\},\left\{\beta_{n, i}\right\}$, $i=1, \ldots,(N+M)$ be sequences in $(0,1)$ such that $\beta_{n, i} \rightarrow \beta_{i} \in(0,1)$ as $n \rightarrow \infty$ for all $i \in$ $\{1, \ldots,(N+M)\}$. Suppose that the conditions $(\mathrm{C} 1)-(\mathrm{C} 6)$ in Theorem 3.1 hold and that the 
sequence $\left\{x_{n}\right\}$ is defined explicitly by the following iterative scheme:

$$
\left\{\begin{array}{l}
\Theta\left(u_{n}, y\right)+h\left(u_{n}, y\right)+\frac{1}{r_{n}}\left\langle y-u_{n}, u_{n}-x_{n}\right\rangle \geq 0, \quad \forall y \in C, \\
y_{n, 1}=\beta_{n, 1} P_{\operatorname{Fix}(T) \cap E P(\Theta, h)} \tilde{S}_{1} u_{n}+\left(1-\beta_{n, 1}\right) u_{n}, \\
y_{n, i}=\beta_{n, i} P_{\operatorname{Fix}(T) \cap E P(\Theta, h)} \tilde{S}_{i} u_{n}+\left(1-\beta_{n, i}\right) y_{n, i-1}, \quad i=2, \ldots, M, \\
\bar{y}_{n, j}=\bar{\beta}_{n, j} P_{C}\left(I-\lambda A_{j}\right) u_{n}+\left(1-\bar{\beta}_{n, j}\right) \bar{y}_{n, j-1}, \quad j=1, \ldots, N, \\
x_{n+1}=P_{C}\left[\lambda_{n} \gamma\left(\alpha_{n} f\left(x_{n}\right)+\left(1-\alpha_{n}\right) S x_{n}\right)+\left(I-\lambda_{n} \mu F\right) T \bar{y}_{n, N}\right], \quad n \geq 1,
\end{array}\right.
$$

where $\bar{y}_{n, 0}=y_{n, M}$ and $\bar{\beta}_{n, j}=\beta_{n, M+j}, j=1, \ldots, N$. In particular, if $f \equiv 0$, then (4.4) reduces to the following iterative scheme:

$$
\left\{\begin{array}{l}
\Theta\left(u_{n}, y\right)+h\left(u_{n}, y\right)+\frac{1}{r_{n}}\left\langle y-u_{n}, u_{n}-x_{n}\right\rangle \geq 0, \quad \forall y \in C, \\
y_{n, 1}=\beta_{n, 1} P_{\operatorname{Fix}(T) \cap E P(\Theta, h)} \tilde{S}_{1} u_{n}+\left(1-\beta_{n, 1}\right) u_{n}, \\
y_{n, i}=\beta_{n, i} P_{\operatorname{Fix}(T) \cap E P(\Theta, h)} \tilde{S}_{i} u_{n}+\left(1-\beta_{n, i}\right) y_{n, i-1}, \quad i=2, \ldots, M, \\
\bar{y}_{n, j}=\bar{\beta}_{n, j} P_{C}\left(I-\lambda A_{j}\right) u_{n}+\left(1-\bar{\beta}_{n, j}\right) \bar{y}_{n, j-1}, \quad j=1, \ldots, N, \\
x_{n+1}=P_{C}\left[\lambda_{n}\left(1-\alpha_{n}\right) \gamma S x_{n}+\left(I-\lambda_{n} \mu F\right) T \bar{y}_{n, N}\right], \quad n \geq 1 .
\end{array}\right.
$$

Then the following assertions hold.

(a) Let $\left\{x_{n}\right\}$ be a sequence generated by the scheme (4.4), then $\left\{x_{n}\right\}$ converges strongly to the point $x^{*} \in \Omega$ which is a unique solution of Problem 4.1.

(b) Let $\left\{x_{n}\right\}$ be a sequence generated by the scheme (4.5), then $\left\{x_{n}\right\}$ converges strongly to a unique solution $x^{*} \in \Omega$ of the following system of variational inequalities:

$$
\begin{cases}\left\langle F x^{*}, x-x^{*}\right\rangle \geq 0, & \forall x \in \Omega, \\ \left\langle(\mu F-\gamma S) x^{*}, y-x^{*}\right\rangle \geq 0, & \forall y \in \Omega .\end{cases}
$$

Now, we consider another more general mixed problem.

Problem 4.2 Let $F: C \rightarrow H$ be $\kappa$-Lipschitzian and $\eta$-strongly monotone on $C, f: C \rightarrow H$ be a $\rho$-contraction with a coefficient $\rho \in[0,1)$ and $S, T$ be nonexpansive self-mappings on $C$. Let $0<\mu<2 \eta / \kappa^{2}$ and $0<\gamma \leq \tau$, where $\tau=1-\sqrt{1-\mu\left(2 \eta-\mu \kappa^{2}\right)}$. Assume that $\Theta, h: C \times C \rightarrow \mathbf{R}$ are two bifunctions. Then the objective is to find $x^{*} \in \Xi$ such that

$$
\left\langle(\mu F-\gamma f) x^{*}, x-x^{*}\right\rangle \geq 0, \quad \forall x \in \Xi
$$

where $\Xi$ denotes the solution set of the following hierarchical variational inequality problem (HVIP) of finding $z^{*} \in \Omega$ such that

$$
\left\langle(\mu F-\gamma S) z^{*}, z-z^{*}\right\rangle \geq 0, \quad \forall z \in \Omega
$$

with $\Omega=\operatorname{Fix}(T) \cap(S V I P) \cap E P(\Theta, h) \neq \emptyset$.

Utilizing Theorem 3.2, we get the following result. 
Theorem 4.2 Let $0<\lambda \leq 2 \alpha$. Let $\left\{\lambda_{n}\right\},\left\{\alpha_{n}\right\},\left\{\beta_{n, i}\right\}, i=1, \ldots,(N+M)$ be sequences in $(0,1)$ such that $\beta_{n, i} \rightarrow \beta_{i} \in(0,1)$ as $n \rightarrow \infty$ for all $i \in\{1, \ldots,(N+M)\}$. Assume that the solution set $\Xi$ of HVIP (4.8) is nonempty and that the conditions (C1)-(C7) in Theorem 3.2 hold. Then the following assertions hold.

(a) Let $\left\{x_{n}\right\}$ be a sequence generated by the scheme (4.4) such that $\omega_{w}\left(x_{n}\right) \subset \Xi$, then $\left\{x_{n}\right\}$ converges strongly to the point $x^{*} \in \Omega$ which is a unique solution of Problem 4.2.

(b) Let $\left\{x_{n}\right\}$ be a sequence generated by the scheme (4.5) such that $\omega_{w}\left(x_{n}\right) \subset \Xi$, then $\left\{x_{n}\right\}$ converges strongly to a unique solution $x^{*}$ of the following VIP:

$$
\text { find } x^{*} \in \Xi \text { such that }\left\langle F x^{*}, x-x^{*}\right\rangle \geq 0, \quad \forall x \in \Xi \text {. }
$$

\section{Concluding remarks}

We considered a system of variational inequalities defined over the intersection of the set of solutions of an equilibrium problem, the set of common fixed points of a finite family of nonexpansive mappings, and the solution set of a nonexpansive mapping (Problem 1.2). We also considered a triple hierarchical variational inequality problem, that is, a variational inequality problem defined over a set of solutions of another variational inequality problem which is defined over the intersection of the set of solutions of an equilibrium problem, the set of common fixed points of a finite family of nonexpansive mappings, and the solution set of a nonexpansive mapping (Problem 1.3). The nontrivial examples of Problems 1.2 and 1.3 are also given in Section 4. We combined the one-step iterative method proposed in [29] and the multi-step iterative method given in [27] to propose a multi-step hybrid viscosity method that generates a sequence via an explicit iterative algorithm. It is worth pointing out that the one-step iterative method given in [29] combines the regularization method, the hybrid steepest-descent method and the projection method, and that the multi-step iterative method given in [27] involves the Ishikawa-type iterative method and the viscosity approximation method. Moreover, it is also proven that under two different pools of suitable conditions such a sequence converges strongly to a unique solution of Problem 1.2 and to a unique solution of Problem 1.3, respectively.

Competing interests

The authors declare that they have no competing interests.

Authors' contributions

All authors participated in the design of this work and performed equally. All authors read and approved the final manuscript.

\section{Author details}

'Department of Mathematics, King Abdulaziz University, Jeddah, 21589, Saudi Arabia. ${ }^{2}$ Department of Mathematics, Scientific Computing Key Laboratory of Shanghai Universities, Shanghai Normal University, Shanghai, 200234, China.

${ }^{3}$ Department of Mathematics, Aligarh Muslim University, Aligarh, 202 002, India.

\section{Acknowledgements}

This paper was funded by the Deanship of Scientific Research (DSR), King Abdulaziz University, Jeddah under the grant No. 107-130-D1432. The authors, therefore, acknowledge with thanks technical and financial support of the DSR. The authors would also like to thank the referees for careful reading of the manuscript.

Received: 18 April 2012 Accepted: 4 October 2012 Published: 24 October 2012

\section{References}

1. Mainge, P-E, Moudafi, A: Strong convergence of an iterative method for hierarchical fixed-points problems. Pac. J. Optim. 3(3), 529-538 (2007)

2. Moudafi, A, Mainge, P-E: Towards viscosity approximations of hierarchical fixed-point problems. Fixed Point Theory Appl. 2006, Art. ID 95453 (2006) 
3. Combettes, PL: A block-iterative surrogate constraint splitting method for quadratic signal recovery. IEEE Trans. Signal Process. 51(7), 1771-1782 (2003)

4. liduka, H: Fixed point optimization algorithm and its application to power control in CDMA data networks. Math. Program. (2010). doi:10.1007/s10107-010-0427-x

5. Slavakis, K, Yamada, I: Robust wideband beamforming by the hybrid steepest descent method. IEEE Trans. Signal Process. 55, 4511-4522 (2007)

6. Cabot, A: Proximal point algorithm controlled by a slowly vanishing term: applications to hierarchical minimization. SIAM J. Optim. 15, 555-572 (2005)

7. Ceng, LC, Khan, AR, Ansari, QH, Yao, JC: Viscosity approximation methods for strongly positive and monotone operators. Fixed Point Theory 10(1), 35-71 (2009)

8. Cianciaruso, F, Colao, V, Muglia, L, Xu, HK: On an implicit hierarchical fixed point approach to variational inequalities. Bull. Aust. Math. Soc. 80(1), 117-124 (2009)

9. liduka, $\mathrm{H}$ : A new iterative algorithm for the variational inequality problem over the fixed point set of a firmly nonexpansive mapping. Optimization 59, 873-885 (2010)

10. liduka, H, Yamada, I: A use of conjugate gradient direction for the convex optimization problem over the fixed point set of a nonexpansive mapping. SIAM J. Optim. 19, 1881-1893 (2009)

11. Marino, G, Xu, HK: Explicit hierarchical fixed point approach to variational inequalities. J. Optim. Theory Appl. 149(1), 61-78 (2011)

12. Moudafi, A: Krasnoselski-Mann iteration for hierarchical fixed-point problems. Inverse Probl. 23, 1635-1640 (2007)

13. Xu, HK: An iterative approach to quadratic optimization. J. Optim. Theory Appl. 116(3), 659-678 (2003)

14. Xu, HK: Viscosity methods for hierarchical fixed point approach to variational inequalities. Taiwan. J. Math. 14(2), 463-478 (2010)

15. Yamada, I, Ogura, N: Hybrid steepest descent method for the variational inequality problem over the fixed point set of certain quasi-nonexpansive mappings. Numer. Funct. Anal. Optim. 25, 619-655 (2004)

16. Yamada, I, Ogura, N, Shirakawa, N: A numerically robust hybrid steepest descent method for the convexly constrained generalized inverse problems. In: Inverse Problems, Image Analysis, and Medical Imaging. Contemp. Math., vol. 313, pp. 269-305. Am. Math. Soc., Providence (2002)

17. Yao, Y, Liou, Y-C: Weak and strong convergence of Krasnoselski-Mann iteration for hierarchical fixed point problems. Inverse Probl. 24(1), Art. ID 015015 (2008)

18. Yao, Y, Chen, R, Xu, HK: Schemes for finding minimum-norm solutions of variational inequalities. Nonlinear Anal. 72 3447-3456 (2010)

19. Yao, Y, Liou, YC, Marino, G: Two-step iterative algorithms for hierarchical fixed point problems and variational inequality problems. J. Appl. Math. Comput. 31(1-2), 433-445 (2009)

20. Blum, E, Oettli, W: From optimization and variational inequalities to equilibrium problems. Math. Stud. 63(1-4), 123-145 (1994)

21. Oettli, W: A remark on vector-valued equilibria and generalized monotonicity. Acta Math. Vietnam. 22, $215-221$ (1997)

22. Combettes, PL, Hirstoaga, SA: Equilibrium programming in Hilbert spaces. J. Nonlinear Convex Anal. 6(1), 117-136 (2005)

23. Moudafi, A: Weak convergence theorems for nonexpansive mappings and equilibrium problems. J. Nonlinear Convex Anal. 9(1), 37-43 (2008)

24. Ceng, LC, Yao, JC: A hybrid iterative scheme for mixed equilibrium problems and fixed point problems. J. Comput. Appl. Math. 214(1), 186-201 (2008)

25. Peng, JW: Iterative algorithms for mixed equilibrium problems, strictly pseudo-contractions and monotone mappings. J. Optim. Theory Appl. 144(1), 107-119 (2010)

26. Peng, JW, Yao, JC: A new hybrid-extragradient method for generalized mixed equilibrium problems, fixed point problems and variational inequality problems. Taiwan. J. Math. 12(6), 1401-1432 (2008)

27. Marino, G, Muglia, L, Yao, Y: Viscosity methods for common solutions of equilibrium and variational inequality problems via multi-step iterative algorithms and common fixed points. Nonlinear Anal. 75, 1787-1798 (2012)

28. Cianciaruso, F, Marino, G, Muglia, L, Yao, Y: A hybrid projection algorithm for finding solutions of mixed equilibrium problem and variational inequality problem. Fixed Point Theory Appl. 2010, Art. ID 383740 (2010)

29. Ceng, LC, Ansari, QH, Yao, JC: Iterative methods for triple hierarchical variational inequalities in Hilbert spaces. J. Optim. Theory Appl. 151, 489-512 (2011)

30. Luo, ZQ, Pang, JS, Ralph, D: Mathematical Programs with Equilibrium Constraints. Cambridge University Press, New York (1996)

31. Outrata, J, Kocvara, M, Zowe, J: Nonsmooth Approach to Optimization Problems with Equilibrium Constraints. Kluwer Academic Press, Dordrecht (1998)

32. liduka, $\mathrm{H}$ : Strong convergence for an iterative method for the triple-hierarchical constrained optimization problem. Nonlinear Anal. 71, 1292-1297 (2009)

33. liduka, $\mathrm{H}$ : Iterative algorithm for solving triple-hierarchical constrained optimization problem. J. Optim. Theory Appl. $148,580-592(2011)$

34. Xu, HK, Kim, TH: Convergence of hybrid steepest-descent methods for variational inequalities. J. Optim. Theory Appl. 119(1), 185-201 (2003)

35. Ceng, LC, Petrusel, A, Yao, JC: Strong convergence theorems of averaging iterations of nonexpansive nonself-mappings in Banach spaces. Fixed Point Theory 8(2), 219-236 (2007)

36. Colao, V, Marino, G, Xu, HK: An iterative method for finding common solutions of equilibrium and fixed point problems. J. Math. Anal. Appl. 344(1), 340-352 (2008)

37. Takahashi, W, Toyoda, M: Weak convergence theorems for nonexpansive mappings and monotone operators. J. Optim. Theory Appl. 118(2), 417-428 (2003) 